\title{
EXISTENCE OF GLOBAL CLASSICAL AND WEAK SOLUTIONS TO A PRION EQUATION WITH POLYMER JOINING
}

\author{
ELENA LEIS AND CHRISTOPH WALKER
}

\begin{abstract}
AвSTRACT. We consider a nonlinear integro-differential equation for prion proliferation that includes prion polymerization, polymer splitting, and polymer joining. The equation can be written as a quasilinear Cauchy problem. For bounded reaction rates we prove global existence and uniqueness of classical solutions by means of evolution operator theory. We also prove global existence of weak solutions for unbounded reaction rates by a compactness argument.
\end{abstract}

\section{INTRODUCTION}

Prions are misfolded proteins and are regarded as the infectious agent of fatal diseases known as TSE's including BSE of cattle, new variant Creutzfeldt-Jakob of human, and Scrapie of sheep. Prions seem to be capable of proliferation despite lacking DNA and RNA. In this article we focus on a mathematical model introduced in [8] for nucleated polymerization which is a theory describing the replication of prions. According to this theory, infectious $\operatorname{Pr} P^{S c}$ prions are thought to be a polymer form of a normal protein monomer $\operatorname{Pr} P^{C}$. Infectious polymers build bonds involving several thousands of monomer units by attaching non-infectious $\operatorname{Pr} P^{C}$ monomers and converting them to the infectious form. Prions are very stable but can also split into smaller polymers. Usually, this produces again two infectious $\operatorname{Pr} P^{S c}$ polymers. However, decay products below a critical size $y_{0}>0$ are assumed to disintegrate instantaneously into $\operatorname{Pr} P^{C}$ monomers. Moreover, two infectious polymers can also join and form longer polymers. We refer to $[8,9,15,16]$ and the references therein for more detailed information on the biological background and on the mechanism of nucleated polymerization.

The biological processes of polymerization, polymer joining, and polymer splitting can be described by a coupled system consisting of an ordinary differential equation for the number of $\operatorname{Pr} P^{C}$ monomers $v(t) \geq 0$ and an integro-differential equation for the density distribution function $u=u(t, y) \geq 0$ for $\operatorname{Pr} P^{S c}$ polymers of size $y>y_{0}$. The monomer equation is

$$
\begin{aligned}
v^{\prime}(t)= & \lambda-\gamma v(t)-\frac{v(t)}{1+\nu \int_{y_{0}}^{\infty} u(t, z) z \mathrm{~d} z} \int_{y_{0}}^{\infty} \tau(y) u(t, y) \mathrm{d} y \\
& +2 \int_{y_{0}}^{\infty} u(t, y) \beta(y) \int_{0}^{y_{0}} z \kappa(z, y) \mathrm{d} z \mathrm{~d} y
\end{aligned}
$$

and the polymer equation is

$$
\partial_{t} u(t, y)+\frac{v(t)}{1+\nu \int_{y_{0}}^{\infty} u(t, z) z \mathrm{~d} z} \partial_{y}(\tau(y) u(t, y))=L[u(t)](y)+Q[u(t), u(t)](y)
$$

for $t>0$ and $y \in Y:=\left(y_{0}, \infty\right)$ involving a linear part $L$ with

$$
L[u](y):=-(\mu(y)+\beta(y)) u(y)+2 \int_{y}^{\infty} \beta(z) \kappa(y, z) u(z) \mathrm{d} z
$$

Date: February 12, 2018.

Key words and phrases. Prions, polymer joining, classical and weak solutions, evolution operators. 
and a bilinear part $Q$ with

$$
Q[u, w](y):=\mathbf{1}_{\left[y>2 y_{0}\right]} \int_{y_{0}}^{y-y_{0}} \eta(y-z, z) u(y-z) w(z) \mathrm{d} z-2 u(y) \int_{y_{0}}^{\infty} \eta(z, y) w(z) \mathrm{d} z .
$$

The equations are supplemented with the boundary condition

$$
u\left(t, y_{0}\right)=0, \quad t>0
$$

and the initial values

$$
v(0)=v^{0}, \quad u(0, y)=u^{0}(y), \quad y \in\left(y_{0}, \infty\right) .
$$

According to the right-hand side of the ordinary differential equation (1.1) the number of monomers is increased by a constant background source $\lambda$ and if a $\operatorname{Pr} P^{S c}$ polymer of any size $y>y_{0}$ decays at a rate $\beta(y)$ into at least one daughter polymer of size $z \leq y_{0}$, which is assumed to disintegrate instantaneously into monomers only. The probability (density) for this event is denoted by $\kappa(z, y)$. The number of $\operatorname{Pr} P^{C}$ monomers decreases by metabolic degradation with rate $\gamma$ and if monomers are attached to a $\operatorname{Pr} P^{S c}$ polymer of size $y>y_{0}$ at rate $\tau(y)$. Accordingly, equation (1.2) for $u$ involves a nonlinear polymerization term

$$
\frac{v(t)}{1+\nu \int_{y_{0}}^{\infty} u(t, z) z \mathrm{~d} z} \partial_{y}(\tau(y) u(y)) .
$$

If $\nu>0$ there is a saturation effect when the number $\int_{y_{0}}^{\infty} u(t, z) z \mathrm{~d} z$ of monomers within the infectious polymers becomes large resulting in less lengthening overall. The right-hand side of (1.2) reflects that polymers of size $y>y_{0}$ disappear due to metabolic degradation with rate $\mu(y)$, by splitting with rate $\beta(y)$, or if they join with another polymer. Also, polymers of size $y>y_{0}$ can be produced by the decay of a larger polymer or if two smaller polymers join. Thus, equation (1.2) is reminiscent of the continuous coagulationfragmentation equation known from physics (see e.g. [6,11] and the references therein).

When polymer joining is neglected, that is, $\eta \equiv 0$, (1.1)-(1.4) and variants thereof were investigated in [5, 9, 13, 18, 19, 21]. More precisely, assuming that the kernels have the particular form

$$
\tau \equiv \mathrm{const}, \quad \mu \equiv \mathrm{const}, \quad \beta(y)=\beta y, \quad \kappa(z, y)=\frac{1}{y},
$$

(1.1)-(1.2) can be integrated and a closed system of ordinary differential equations for the unknowns $v$, $\int_{Y} u(t, y) \mathrm{d} y$, and $\int_{Y} y u(t, y) \mathrm{d} y$ can be obtained which possesses a unique global solution as shown in [9, 18] (for $\nu=0$ ). In these articles also stability of equilibria were studied. Note that in this case the solution $v$ to (1.1) is then determined and thus (1.1)-(1.2) decouples leaving one with a non-local, but linear integro-differential equation for $u$ for which well-posedness and asymptotic stability of equilibria were shown in [5]. For $\eta \equiv 0$, well-posedness of global classical and weak solutions to the coupled system (1.1)(1.4) without assuming (1.5) was established in [13, 19,21]. Let us also point out that certain qualitative aspects of (1.1)-(1.2) (still with $\eta \equiv 0$ ) were investigated e.g. in [1-3, 7]. The model with polymer joining was introduced in [8]. Assuming (1.5) and $\eta \equiv$ const, equations (1.1)-(1.2) can again be integrated to a system of ordinary differential equations for which global well-posedness and stability of equilibria was studied in [8].

The main contribution of this article is the inclusion of the bilinear polymer joining part $Q[u, u]$. We prove existence and uniqueness of global classical solutions as in [19,21] and existence of global weak solutions as in [13]. Note that this does not seem to be straightforward since the linear part $L[u]$ can be considered as a perturbation of the first order polymerization term and thus, for $\eta \equiv 0$ (i.e. $Q \equiv 0$ ), equation (1.2) is homogeneous and considerably simpler to handle, see [19, 21]. Including $Q$ requires additional arguments and the proofs - in particular for classical solutions - become more involved as we shall see later on (see the remarks at the end of Subsection 3.1). 


\section{MAin Results}

Throughout this article we assume that

$$
\nu, \lambda, \gamma \geq 0
$$

The splitting kernel $\kappa \geq 0$ is a measurable function defined on $\mathcal{K}:=\left\{(z, y) ; y_{0}<y<\infty, 0<z<y\right\}$ satisfying the symmetry condition

$$
\kappa(z, y)=\kappa(y-z, y), \quad(z, y) \in \mathcal{K},
$$

and is normalized according to

$$
2 \int_{0}^{y} z \kappa(z, y) \mathrm{d} z=y, \quad \text { a.a. } y \in Y .
$$

Thus, splitting conserves the number of monomers and (2.2), 2.3) imply

$$
\int_{0}^{y} \kappa(z, y) \mathrm{d} z=1, \quad \text { a.a. } y \in Y .
$$

The polymer joining kernel $\eta$ is symmetric, that is,

$$
\eta(y, z)=\eta(z, y), \quad y, z \in Y .
$$

We then remark that (2.5) (formally) implies the identities

$$
\begin{aligned}
\int_{y_{0}}^{\infty} \varphi(y) L[u](y) \mathrm{d} y= & -\int_{y_{0}}^{\infty} \varphi(y) \mu(y) u(y) \mathrm{d} y \\
& +\int_{y_{0}}^{\infty} u(y) \beta(y)\left(-\varphi(y)+2 \int_{y_{0}}^{y} \varphi(z) \kappa(z, y) \mathrm{d} z\right) \mathrm{d} y
\end{aligned}
$$

and

$$
\int_{y_{0}}^{\infty} \varphi(y) Q[u, u](y) \mathrm{d} y=\int_{y_{0}}^{\infty} \int_{y_{0}}^{\infty}(\varphi(y+z)-\varphi(y)-\varphi(z)) \eta(y, z) u(y) u(z) \mathrm{d} z \mathrm{~d} y .
$$

In particular, with $\varphi(y)=y$ we obtain from (2.3) that a solution $(v, u)$ to (1.1)-(1.4) satisfies (formally) the monomer balance law

$$
\begin{aligned}
v(t)+ & \int_{y_{0}}^{\infty} y u(t, y) \mathrm{d} y-v^{0}-\int_{y_{0}}^{\infty} y u^{0}(y) \mathrm{d} y \\
& =\lambda t-\gamma \int_{0}^{t} v(s) \mathrm{d} s-\int_{0}^{t} \int_{y_{0}}^{\infty} y \mu(y) u(s, y) \mathrm{d} y \mathrm{~d} s
\end{aligned}
$$

at time $t$. Thus, the number of monomers only changes due to natural production or metabolic degradation. This relation turns out to be crucial with respect to the existence of global solutions as it provides suitable a priori estimates. This, however, seems to be the only available information.

In the following we use $L_{1}(Y, y \mathrm{~d} y)$ as a state space for the population density $u$ and denote its positive cone by $L_{1}^{+}(Y, y \mathrm{~d} y)$. This allows us to keep track of the biologically important quantities

$$
\int_{y_{0}}^{\infty} u(t, y) \mathrm{d} y \quad \text { and } \quad \int_{y_{0}}^{\infty} u(t, y) y \mathrm{~d} y
$$

of all $\operatorname{Pr} P^{S c}$ polymers respectively $\operatorname{Pr} P^{C}$ monomers forming those polymers. 
2.1. Classical Solutions for Bounded Kernels. We consider first bounded kernels $\mu, \beta, \eta$, and $\tau$. More precisely, we let

$$
\mu, \beta \in L_{\infty}^{+}(Y), \quad \eta \in B C^{1}\left(Y \times Y, \mathbb{R}^{+}\right)
$$

and

$$
\tau \in B C^{1}\left(Y, \mathbb{R}^{+}\right), \quad \tau(y) \geq \tau_{0}, \quad y \in\left(y_{0}, \infty\right),
$$

for some constant $\tau_{0}>0$. The boundedness (2.9) of the kernels in particular imply that the operators $L$ and $Q$ are bounded and linear, respectively, bilinear operators from $L_{1}$ into itself. Using this we can proof the existence and uniqueness of global classical solutions:

Theorem 2.1. Suppose (2.1)-(2.3), (2.5), (2.9), and (2.10). Then, given any initial values $v^{0}>0$ and $u^{0} \in L_{1}^{+}(Y, y \mathrm{~d} y)$ with $\partial_{y} u^{0} \in L_{1}(Y, y \mathrm{~d} y)$ and $u^{0}\left(y_{0}\right)=0$, there exists a unique global classical solution $(v, u)$ to 1.1 -1.4 such that $v \in C^{1}\left(\mathbb{R}^{+}\right)$and $u \in C^{1}\left(\mathbb{R}^{+}, L_{1}(Y, y \mathrm{~d} y)\right)$ with $\partial_{y} u \in C\left(\mathbb{R}^{+}, L_{1}(Y, y \mathrm{~d} y)\right)$. This solution is positive, that is, $v(t)>0, u(t) \in L_{1}^{+}(Y, y \mathrm{~d} y)$ for $t \geq 0$, and it is monomer preserving, that is, it satisfies the balance law (2.8).

To prove Theorem 2.1 we shall write (1.1)-(1.4) as a quasilinear hyperbolic Cauchy problem for $u$, where the nonlinear transport term generates an evolution operator in the phase space $L_{1}(Y, y \mathrm{~d} y)$ and the linear part $L$ can be considered as a linear perturbation thereof. Owing to the bilinear operator $Q$ the Cauchy problem is, in contrast to [19,21], no longer homogeneous. We shall see in Section 3 that the fixed point argument to solve this Cauchy problem thus becomes more involved and has to be performed twice (in different function spaces) to cope with the lacking regularization of the hyperbolic evolution operator and the nonlinearities stemming from polymer joining.

2.2. Weak Solutions for Unbounded Kernels. The assumptions (2.9), 2.10) that the kernels are bounded seem to be rather strong from a biological point of view since they exclude e.g. splitting rates as in (1.5). In order to include unbounded kernels we weaken the notion of a solution.

Definition 2.2. Given $v^{0}>0$ and $u^{0} \in L_{1}^{+}(Y, y \mathrm{~d} y)$ we call a pair $(v, u)$ a (monomer preserving) global weak solution to (1.1)-(1.4) provided the following conditions are satisfied:

(i) $v \in C^{1}\left(\mathbb{R}^{+}\right)$is a non-negative solution to 1.1 ,

(ii) $u \in L_{\infty, \operatorname{loc}}\left(\mathbb{R}^{+}, L_{1}^{+}\right)$is a weak solution to 1.2 , that is, it satisfies for all $t>0$

$$
\begin{aligned}
& {[(s, y) \mapsto(\mu(y)+\beta(y)) u(s, y)] \in L_{1}((0, t) \times Y)} \\
& {[(s, y, z) \mapsto \eta(y, z) u(s, y) u(s, z)] \in L_{1}((0, t) \times Y \times Y)}
\end{aligned}
$$

and

$$
\begin{aligned}
\int_{y_{0}}^{\infty} \varphi(y) & u(t, y) \mathrm{d} y-\int_{y_{0}}^{\infty} \varphi(y) u^{0}(y) \mathrm{d} y \\
= & \int_{0}^{t} \frac{v(s)}{1+\nu\|u(s)\|_{L_{1}(Y, y \mathrm{~d} y)}} \int_{y_{0}}^{\infty} \varphi^{\prime}(y) \tau(y) u(s, y) \mathrm{d} y \mathrm{~d} s \\
& -\int_{0}^{t} \int_{y_{0}}^{\infty} \varphi(y) \mu(y) u(s, y) \mathrm{d} y \mathrm{~d} s \\
& +\int_{0}^{t} \int_{y_{0}}^{\infty} u(s, y) \beta(y)\left(-\varphi(y)+2 \int_{y_{0}}^{y} \varphi(z) \kappa(z, y) \mathrm{d} z\right) \mathrm{d} y \mathrm{~d} s \\
& +\int_{0}^{t} \int_{y_{0}}^{\infty} \int_{y_{0}}^{\infty}(\varphi(y+z)-\varphi(y)-\varphi(z)) \eta(y, z) u(s, y) u(s, z) \mathrm{d} z \mathrm{~d} y \mathrm{~d} s
\end{aligned}
$$

for any test function $\varphi \in W_{\infty}^{1}(Y)$,

(iii) the balance law (2.8) holds. 
The weak formulation in (ii) above is obtained by testing (1.2) against $\varphi$ and using the identity (2.7). To prove the existence of a weak solution we do no longer need bounded kernels but rather impose certain growth conditions. More precisely, we suppose that

$$
\mu, \beta \in L_{\infty, \mathrm{loc}}^{+}(Y)
$$

and

$$
\tau \in C\left(\left[y_{0}, \infty\right)\right) \quad \text { with } \quad \tau_{0} \leq \tau(y) \leq \tau_{*} y, \quad y \geq y_{0},
$$

for some constants $\tau_{0}, \tau_{*}>0$. The measurable function $\kappa$ is supposed to satisfy (2.2), (2.3) and given any $R>y_{0}$ it holds that

$$
\lim _{\delta \rightarrow 0} \sup _{\substack{E \subset\left(y_{0}, R\right) \\|E| \leq \delta}} \operatorname{ess-sup}_{y \in\left(y_{0}, R\right)} \beta(y) \int_{y_{0}}^{y} \mathbf{1}_{E}(z) \kappa(z, y) \mathrm{d} z=0,
$$

where $|E|$ denotes the Lebesgue measure of a measurable set $E \subset Y$. Condition (2.15) is used later on to guarantee the uniform integrability of a sequence of approximative solutions. Furthermore, let there be $y_{1} \in Y$ and $\delta_{1}>0$ such that

$$
\int_{y_{1}}^{y}\left(1-\frac{z}{y}\right) \kappa(z, y) \mathrm{d} z \geq \delta_{1}, \quad y \geq 2 y_{1} .
$$

The polymer joining kernel $\eta$ shall be a continuous function $Y \times Y \rightarrow \mathbb{R}^{+}$satisfying (2.5) and

$$
\eta(y, z) \leq K\left(y^{\alpha} z^{\rho}+y^{\rho} z^{\alpha}\right) \quad(y, z) \in Y \times Y,
$$

for some constant $K \geq 1$ and a pair of numbers $(\alpha, \rho)$ with

$$
0 \leq \alpha \leq \rho \leq 1, \quad \theta:=\alpha+\rho \in[0,2],
$$

ensuring the integrability of $Q$. In case that $\theta \in(1,2]$ we additionally require that there are $B>0$, $\zeta>\theta-1$, and $0<a<1$ such that

$$
\beta(y) \geq B y^{\zeta}, \quad 2 \int_{y_{0}}^{y} z \kappa(z, y) \mathrm{d} z \leq a y, \quad y \in Y .
$$

The imposed conditions are similar as in [13, 19,21]. We remark that a class of examples for $\kappa$ is obtained when of the form

$$
\kappa(z, y)=\frac{1}{y} k_{0}\left(\frac{z}{y}\right), \quad y>y_{0}, \quad 0<z<y,
$$

with a non-negative integrable function $k_{0}$ defined on $(0,1)$ satisfying

$$
k_{0}(y)=k_{0}(1-y), \quad y \in(0,1), \quad \int_{0}^{1} k_{0}(y) \mathrm{d} y=1 .
$$

One then readily checks that the conditions (2.2), (2.3), (2.15), and (2.16) hold. In particular, for $k_{0} \equiv 1$ one has

as considered in [5,8,9].

$$
\kappa(z, y)=\frac{1}{y}, \quad y>y_{0}, \quad 0<z<y,
$$

To state our result on existence of weak solutions we shall use the notation $L_{1, \mathrm{w}}(Y, y \mathrm{~d} y)$ for the space $L_{1}(Y, y \mathrm{~d} y)$ endowed with its weak topology.

Theorem 2.3. Suppose (2.1)-(2.3), (2.5), and (2.13)-(2.18). If $\theta=\alpha+\rho \in(1,2]$, then also suppose (2.19). Let $\left(v_{0}, u_{0}\right)$ with $v^{0}>0$ and $u^{0} \in L_{1}^{+}(Y, y \mathrm{~d} y)$. Then there exists a monomer preserving global weak solution $(v, u)$ in the sense of Definition 2.2 with $u \in C\left(\mathbb{R}^{+}, L_{1, \mathrm{w}}(Y, y \mathrm{~d} y)\right)$. 
The construction of a monomer preserving global weak solution results from a compactness argument. For suitably truncated bounded kernels we first obtain from Theorem 2.1 a sequence $\left(\left(v_{n}, u_{n}\right)\right)_{n \in \mathbb{N}}$ of global classical solutions. We then use the balance law (2.8) and the Dunford-Pettis Theorem to derive compactness of this sequence in the space $C\left([0, T], \mathbb{R} \times L_{1, \mathrm{w}}(Y, y \mathrm{~d} y)\right)$ for any given $T>0$. Finally, we show that any cluster point of the sequence $\left(\left(v_{n}, u_{n}\right)\right)_{n \in \mathbb{N}}$ represents a monomer preserving global weak solution.

The previous compactness argument providing the existence of weak solutions does obviously not lead to uniqueness of such a solution. However, one can give sufficient conditions for uniqueness of weak solutions and thus obtain a well-posedness result for weak solutions [14]. This requires additional integrability properties of weak solutions as stated in the following result:

Proposition 2.4. Let the assumptions of Theorem 2.3 with $\theta=\alpha+\rho \leq 1$ hold. If $u^{0} \in L_{1}^{+}\left(Y, y^{\sigma} \mathrm{d} y\right)$ for some $\sigma \geq 1$, then $u \in L_{\infty, \text { loc }}\left(\mathbb{R}^{+}, L_{1}\left(Y, y^{\sigma} \mathrm{d} y\right)\right)$.

\section{Proof of Theorem 2.1}

This section is dedicated to the existence and uniqueness of global classical solutions for bounded kernels for which we invoke the theory of evolution operators. Throughout we suppose the assumptions stated in Theorem 2.1.

3.1. Preliminaries. The boundedness (2.9) of the kernels implies that the operators $L$ and $Q$ are bounded and linear, respectively, bilinear operators from $L_{1}(Y, y \mathrm{~d} y)$ into itself. More precisely, putting

$$
E_{0}:=L_{1}(Y, y \mathrm{~d} y)
$$

equipped with the norm $\|\cdot\|_{0}:=\|\cdot\|_{L_{1}(Y, y \mathrm{~d} y)}$, denoting its positive cone by $E_{0}^{+}$, and setting

$$
E_{1}:=\left\{u \in E_{0}: \partial_{y}(\tau u) \in E_{0}, u\left(y_{0}\right)=0\right\}
$$

equipped with the norm (see 2.14 )

$$
\|u\|_{1}:=\|u\|_{0}+\left\|\partial_{y}(\tau u)\right\|_{0}, u \in E_{1},
$$

we readily obtain:

Lemma 3.1. (a) The operator $L: E_{0} \rightarrow E_{0}$ is bounded and linear with

$$
\|L[u]\|_{0} \leq c_{*}\left(\|\mu\|_{\infty}+\|\beta\|_{\infty}\right)\|u\|_{0}, \quad u \in E_{0} .
$$

(b) For $j \in\{0,1\}$ the operator $Q: E_{j} \times E_{0} \rightarrow E_{j}$ is bounded and bilinear with

$$
\|Q[u, w]\|_{j} \leq c_{*}\|\eta\|_{\infty}\|u\|_{j}\|w\|_{0}, \quad u \in E_{j}, w \in E_{0} .
$$

It is worthwhile pointing out the property of $Q[\cdot, w]$ mapping $E_{j}$ into itself for both $j=0$ and $j=1$ when $w \in E_{0}$ is fixed. This property is crucial for the existence of classical solutions.

To set the stage for a fixed point formulation of (1.1)-(1.4) we next focus on the polymerization term in (1.2) and recall that it is the generator of a positive evolution operator on $E_{0}$ with domain $E_{1}$. For this we define a diffeomorphism $\Theta: Y \rightarrow(0, \infty)$ by virtue of

$$
\Theta(y):=\int_{y_{0}}^{y} \frac{\mathrm{d} y^{\prime}}{\tau\left(y^{\prime}\right)}, \quad y \in Y
$$

Given $f \in E_{0}$ we put

$$
(\mathcal{W}(t) f)(y):=\mathbf{1}_{[t, \infty)}(\Theta(y)) \frac{\tau\left(\Theta^{-1}(\Theta(y)-t)\right)}{\tau(y)} f\left(\Theta^{-1}(\Theta(y)-t)\right), \quad y \in Y, t \geq 0 .
$$

It then follows from [21] that $\{\mathcal{W}(t) ; t \geq 0\}$ is a strongly continuous positive semigroup on $E_{0}$ with generator $-A$ given by

$$
A u:=\partial_{y}(\tau u), \quad u \in E_{1} .
$$


Moreover, putting $\tau_{*}:=\|\tau\|_{\infty} / y_{0}$ so that $\tau(y) \leq \tau_{*} y, y \in Y$, the estimate

$$
\|\mathcal{W}(t)\|_{\mathcal{L}\left(E_{0}\right)} \leq e^{\tau_{*} t}, \quad t \geq 0
$$

holds and shows that the semigroup is stable in the sense of [17]. Given $T \in(0,1]$ and $R>1$ define $J_{T}:=[0, T]$ and

$$
\mathcal{V}_{T, R}:=\left\{V \in C^{1}\left(J_{T}\right) ; R^{-1} \leq V(t) \leq\|V\|_{C^{1}\left(J_{T}\right)} \leq R\right\} .
$$

Then we introduce for $V \in \mathcal{V}_{T, R}$ the operator

$$
\mathbb{A}_{V}(t) u:=V(t) \partial_{y}(\tau u)-L[u], \quad u \in E_{1}, \quad t \in J_{T},
$$

and recall that $L$ is a bounded operator on $E_{0}$. It was shown in [19,21] analogously to [17, §5] that the stability (3.3) implies that the operator family $\left\{-\mathbb{A}_{V}(t)\right\}_{t \in[0, T]}$ generates an evolution operator on $E_{0}$. More precisely:

Proposition 3.2. Let $R>0, T_{0}>0$, and $0<T \leq T_{0}$ be given. Then $\left\{-\mathbb{A}_{V}(t)\right\}_{t \in[0, T]}$ generates for each $V \in \mathcal{V}_{T, R}$ a unique evolution operator $\mathbb{U}_{V}(t, s), 0 \leq s \leq t \leq T$, in $E_{0}$ enjoying properties $\left(E_{1}\right)-\left(E_{5}\right)$ in [17 §5]. Moreover, there is $\omega_{0}:=\omega_{0}\left(T_{0}, R\right)>0$ such that

$$
\left\|\mathbb{U}_{V}(t, s)\right\|_{\mathcal{L}\left(E_{0}\right)} \leq e^{\omega_{0}(t-s)}, \quad 0 \leq s \leq t \leq T, \quad V \in \mathcal{V}_{T, R},
$$

and

$$
\left\|\mathbb{U}_{V}(t, s)\right\|_{\mathcal{L}\left(E_{1}\right)} \leq \omega_{0}, \quad 0 \leq s \leq t \leq T, \quad V \in \mathcal{V}_{T, R}
$$

and if $V, W \in \mathcal{V}_{T, R}$, then

$$
\left\|\mathbb{U}_{W}(t, s)-\mathbb{U}_{V}(t, s)\right\|_{\mathcal{L}\left(E_{1}, E_{0}\right)} \leq \omega_{0}(t-s)\|W-V\|_{C\left(J_{T}\right)}, \quad 0 \leq s \leq t \leq T .
$$

The $u$ component of a solution $(v, u)$ to (1.1)-(1.4) can then be expressed in the form

$$
u(t)=\mathbb{U}_{V_{u}}(t, 0) u^{0}+\int_{0}^{t} \mathbb{U}_{V_{u}}(t, s) Q[u(s), u(s)] \mathrm{d} s,
$$

where

$$
V_{u}(t)=\frac{v(t)}{1+\nu \int_{y_{0}}^{\infty} z u(t, z) \mathrm{d} z},
$$

which can be regarded as a fixed point equation for $u$. Let us point out that (3.8) guarantees Lipschitz continuity of the evolution operator $\mathbb{U}_{V_{u}}$ with respect to $V_{u}$ only when being considered as an operator from $E_{1}$ to $E_{0}$ while semigroup theory requires the nonlinearity $Q$ to map into $E_{1}$ to guarantee time differentiability of the integral term. To cope with these somewhat antagonizing facts the fixed point argument has to be performed twice, once in $E_{1}$ to ensure time differentiability with regard to classical solutions and once in $E_{0}$ to handle the quasilinear part $V_{u}$ of the problem. As pointed out before, the properties of $Q$ stated in part (b) of Lemma 3.1 are crucial in this respect as we shall see in the next section.

3.2. Local Existence. Let $v^{0}>0$ and $u^{0} \in E_{1} \cap E_{0}^{+}$be given and let $S>0$ be such that

$$
S^{-1} \leq v^{0} \leq S, \quad\left\|u^{0}\right\|_{1} \leq S .
$$

We put

$$
r(S):=2 S \nu\left[\left(S+2 S\|\beta\|_{\infty}+\lambda\right) \frac{\|\tau\|_{\infty}}{y_{0}}+\|\mu\|_{\infty}+\|\beta\|_{\infty}\right]
$$

and then introduce for $\delta \in\{0, \nu\}$ the complete metric space

$$
\begin{array}{r}
Z_{T}^{\delta}:=\left\{u \in C\left(J_{T}, E_{0}^{+}\right) ;\left[t \mapsto \delta\|u(t)\|_{0}\right] \in C^{1}\left(J_{T}\right),\|u(t)\|_{0} \leq 2 S,\right. \\
\left.\left|\frac{\mathrm{d}}{\mathrm{d} t} \delta\|u(t)\|_{0}\right| \leq r(S), t \in J_{T}, u(0)=u^{0}\right\}
\end{array}
$$


equipped with the metric

$$
d_{Z_{T}^{\delta}}(u, w):=\|u-w\|_{C\left(J_{T}, E_{0}\right)}+\delta\|\| u\left\|_{0}-\right\| w\left\|_{0}\right\|_{C^{1}\left(J_{T}\right)}, \quad u, w \in Z_{T}^{\delta} .
$$

Note that

$$
\delta\|u(t)\|_{0}=\delta \int_{y_{0}}^{\infty} y u(t, y) \mathrm{d} y, \quad t \in J_{T},
$$

for $u \in Z_{T}^{\delta}$ and that this term vanishes for $\delta=0$. Let $\bar{u} \in Z_{T}^{\nu}$ be fixed and put

$$
g(\bar{u}(t)):=2 \int_{y_{0}}^{\infty} \bar{u}(t, y) \beta(y) \int_{0}^{y_{0}} z \kappa(z, y) \mathrm{d} z \mathrm{~d} y,
$$

and

$$
p(\bar{u}(t)):=\frac{1}{1+\nu\|\bar{u}(t)\|_{0}} \int_{y_{0}}^{\infty} \tau(y) \bar{u}(t, y) \mathrm{d} y .
$$

Note that both $g(\bar{u})$ and $p(\bar{u})$ are non-negative functions. Consequently, the function $v_{\bar{u}} \in C^{1}\left(J_{T}\right)$, given by

$$
\begin{aligned}
v_{\bar{u}}(t):=\exp & \left(-\gamma t-\int_{0}^{t} p(\bar{u}(\sigma)) \mathrm{d} \sigma\right) v^{0} \\
& +\int_{0}^{t} \exp \left(-\gamma(t-s)-\int_{s}^{t} p(\bar{u}(\sigma)) \mathrm{d} \sigma\right)(\lambda+g(\bar{u}(s))) \mathrm{d} s, \quad t \in J_{T},
\end{aligned}
$$

defines the unique solution to (1.1) with $v_{\bar{u}}(0)=v^{0}$, when $u$ therein is replaced by $\bar{u}$. We then introduce

$$
V_{\bar{u}}(t):=\frac{v_{\bar{u}}(t)}{1+\nu\|\bar{u}(t)\|_{0}}, \quad t \in J_{T}, \quad \bar{u} \in Z_{T}^{\nu} .
$$

Owing to (2.3) and the assumptions on $\beta$ and $\tau$ we have

$$
g(\bar{u}(t)) \leq\|\beta\|_{\infty}\|\bar{u}(t)\|_{0}, \quad p(\bar{u}(t)) \leq \frac{\|\tau\|_{\infty}}{y_{0}}\|\bar{u}(t)\|_{0}
$$

for $0 \leq t \leq T$. Therefore, since $\bar{u} \in Z_{T}^{\nu}$ and

$$
V_{\bar{u}}^{\prime}(t)=\frac{v_{\bar{u}}^{\prime}(t)}{1+\nu\|\bar{u}(t)\|_{0}}-\frac{v_{\bar{u}}(t)}{\left(1+\nu\|\bar{u}(t)\|_{0}\right)^{2}} \nu \frac{\mathrm{d}}{\mathrm{d} t}\|\bar{u}(t)\|_{0}, \quad t \in J_{T},
$$

it readily follows from (3.12) and (1.1) that there exists a constant $R(S)>1$ independent of $T \in(0,1]$ (and $\bar{u}$ ) such that $V_{\bar{u}} \in \mathcal{V}_{T, R(S)}$ for $\bar{u} \in Z_{T}^{\nu}$. Moreover, since

$$
\left|p\left(\bar{u}_{1}(t)\right)-p\left(\bar{u}_{2}(t)\right)\right| \leq \frac{\|\tau\|_{\infty}}{y_{0}}\left\|\bar{u}_{1}(t)-\bar{u}_{2}(t)\right\|_{0}+\frac{\nu\|\tau\|_{\infty}}{y_{0}}\left\|\bar{u}_{1}(t)\right\|_{0}\left|\left\|\bar{u}_{1}(t)\right\|_{0}-\left\|\bar{u}_{2}(t)\right\|_{0}\right|,
$$

formula (3.12) implies that there is a constant $c(S)>0$ independent of $T \in(0,1]$ such that

$$
\left|v_{\bar{u}_{1}}(t)-v_{\bar{u}_{2}}(t)\right| \leq T c(S)\left\|\bar{u}_{1}-\bar{u}_{2}\right\|_{C\left(J_{T}, E_{0}\right)}, \quad 0 \leq t \leq T, \quad \bar{u}_{1}, \bar{u}_{2} \in Z_{T}^{\nu} .
$$

Therefore, from

$$
\left|V_{\bar{u}_{1}}(t)-V_{\bar{u}_{2}}(t)\right| \leq\left|v_{\bar{u}_{1}}(t)-v_{\bar{u}_{2}}(t)\right|+\nu v_{\bar{u}_{2}}(t)||\left|\bar{u}_{1}(t)\left\|_{0}-\right\| \bar{u}_{2}(t) \|_{0}\right|
$$

we obtain, on the one hand,

$$
\left|V_{\bar{u}_{1}}(t)-V_{\bar{u}_{2}}(t)\right| \leq c(S)\left\|\bar{u}_{1}-\bar{u}_{2}\right\|_{C\left(J_{T}, E_{0}\right)}
$$

and, on the other hand since $t \mapsto \nu\left\|u_{j}(t)\right\|_{0}$ is differentiable,

$$
\begin{aligned}
\left|V_{\bar{u}_{1}}(t)-V_{\bar{u}_{2}}(t)\right| & \leq T c(S)\left(\left\|\bar{u}_{1}-\bar{u}_{2}\right\|_{C\left(J_{T}, E_{0}\right)}+\nu\|\| \bar{u}_{1}\left\|_{0}-\right\| \bar{u}_{2}\left\|_{0}\right\|_{C^{1}\left(J_{T}\right)}\right) \\
& =T c(S) d_{Z_{T}^{\nu}}\left(\bar{u}_{1}, \bar{u}_{2}\right)
\end{aligned}
$$


for $0 \leq t \leq T \leq 1$ and $\bar{u}_{1}, \bar{u}_{2} \in Z_{T}^{\nu}$. We then consider for fixed $\bar{u} \in Z_{T}^{\nu}$ and $\hat{u} \in Z_{T}^{0}$ the equation

$$
u^{\prime}+\mathbb{A}_{V_{\bar{u}}}(t) u=Q[u, \hat{u}(t)], \quad t \in J_{T}, \quad u(0)=u^{0},
$$

where the operator

$$
-\mathbb{A}_{V_{\bar{u}}}(t) u=-V_{\bar{u}}(t) \partial_{y}(\tau u)+L[u], \quad u \in E_{1}, \quad t \in J_{T},
$$

is meaningful since $V_{\bar{u}} \in \mathcal{V}_{T, R(S)}$ and thus generates an evolution operator on $E_{0}$ with properties as stated in Proposition 3.2. Note that, for $\bar{u} \in Z_{T}^{\nu}$ and $\hat{u} \in Z_{T}^{0}$ still fixed, the right hand side of (3.17) is a bounded linear operator from $E_{1}$ into itself with respect to $u$ according to Lemma 3.1 (b) which depends continuously on $t$. Standard arguments (e.g. see [17, §5]) then ensure that (3.17) has a unique classical solution

$$
u:=u(\bar{u}, \hat{u}) \in C\left(J_{T}, E_{1}\right) \cap C^{1}\left(J_{T}, E_{0}\right) .
$$

To prove that this solution is non-negative we introduce for technical reasons the constant

$$
\omega:=\frac{4 S}{y_{0}}\|\eta\|_{\infty}+\|\mu+\beta\|_{\infty} .
$$

We then observe that $u$ also solves the problem

$$
w^{\prime}+\left(A_{V_{\bar{u}}}(t)+\omega\right) w=H(t)[w], \quad t \in J_{T}, \quad w(0)=u^{0},
$$

where

$$
-A_{V_{\bar{u}}}(t) w:=-V_{\bar{u}}(t) \partial_{y}(\tau w), \quad w \in E_{1}, \quad t \in J_{T},
$$

generates an evolution operator on $E_{0}$ according to Proposition 3.2 and the bounded operator $H(t) \in$ $\mathcal{L}\left(E_{0}\right)$, given by

$$
H(t)[w]:=Q[w, \hat{u}(t)]+L[w]+\omega w, \quad w \in E_{0},
$$

depends continuously on $t$ and satisfies

$$
H(t)[w] \in E_{0}^{+}, \quad w \in E_{0}^{+}
$$

due to the choice of the constant $\omega$. Recall that the semigroup $\mathcal{W}$ on $E_{0}$ generated by $-\partial_{y}(\tau \cdot)$ is positive. Then clearly $-A_{V_{\bar{u}}}(t)-\omega$ generates a positive semigroup on $E_{0}$ for each $t \in J_{T}$ fixed. The construction of evolution operators (see [17, Theorem 5.3.1] and [21]) entails that the evolution operator generated by $-A_{V_{\bar{u}}}-\omega$ is positive as well. This together with $u^{0} \in E_{0}^{+}$and $(3.20)$ then easily yields that $u(t) \in E_{0}^{+}$for $t \in J_{T}$ (see also the proof of [19, Theorem 3.1]).

Keeping $\bar{u} \in Z_{T}^{\nu}$ still fixed we next show that the mapping $\hat{u} \mapsto \Lambda_{\bar{u}}[\hat{u}]:=u(\bar{u}, \hat{u})$ is a contraction on $Z_{T}^{0}$ for sufficiently small $T \in(0,1]$. For this note (setting $E_{1}^{+}:=E_{0}^{+} \cap E_{1}$ ) that

$$
\Lambda_{\bar{u}}[\hat{u}]=u(\bar{u}, \hat{u}) \in C\left(J_{T}, E_{1}^{+}\right) \cap C^{1}\left(J_{T}, E_{0}\right)
$$

satisfies

$$
u^{\prime}+\mathbb{A}_{V_{\bar{u}}}(t) u=Q[u, \hat{u}(t)], \quad t \in J_{T}, \quad u(0)=u^{0},
$$

and can thus be written as

$$
\Lambda_{\bar{u}}[\hat{u}](t)=\mathbb{U}_{V_{\bar{u}}}(t, 0) u^{0}+\int_{0}^{t} \mathbb{U}_{V_{\bar{u}}}(t, s) Q\left[\Lambda_{\bar{u}}[\hat{u}](s), \hat{u}(s)\right] \mathrm{d} s, \quad t \in J_{T},
$$

where the evolution operator $\mathbb{U}_{V_{\bar{u}}}(t, s)$ enjoys the properties stated in Proposition 3.2 with $\omega_{0}:=\omega_{0}(1, R(S))$. Consequently, it follows from Proposition 3.2 and Lemma 3.1 that

$$
\begin{aligned}
\left\|\Lambda_{\bar{u}}[\hat{u}](t)\right\|_{k} & \leq\left\|\mathbb{U}_{V_{\bar{u}}}(t, 0)\right\|_{\mathcal{L}\left(E_{k}\right)}\left\|u^{0}\right\|_{k}+\int_{0}^{t}\left\|\mathbb{U}_{V_{\bar{u}}}(t, s)\right\|_{\mathcal{L}\left(E_{k}\right)}\left\|Q\left[\Lambda_{\bar{u}}[\hat{u}](s), \bar{u}(s)\right]\right\|_{k} \mathrm{~d} s \\
& \leq e^{\omega_{0}(T(1-k)+k)}\left\|u^{0}\right\|_{k}+2 S c_{*}\|\eta\|_{\infty} e^{\omega_{0}} \int_{0}^{t}\left\|\Lambda_{\bar{u}}[\hat{u}](s)\right\|_{k} \mathrm{~d} s
\end{aligned}
$$


for $k=0,1$ and $0 \leq t \leq T \leq 1$. Thus, taking $k=0$ in (3.23) and recalling (3.9), Gronwall's lemma entails that

$$
\left\|\Lambda_{\bar{u}}[\hat{u}](t)\right\|_{0} \leq 2 S, \quad 0 \leq t \leq T,
$$

provided that $T=T(S) \in(0,1]$ is chosen sufficiently small. This shows that $\Lambda_{\bar{u}}[\hat{u}]=u(\bar{u}, \hat{u}) \in Z_{T}^{0}$ for $\hat{u} \in Z_{T}^{0}$. Moreover, taking $k=1$ in (3.23) Gronwall's lemma also implies that

$$
\left\|\Lambda_{\bar{u}}[\hat{u}](t)\right\|_{1} \leq m(S), \quad 0 \leq t \leq T,
$$

for some constant $m(S)>0$. To show that the mapping $\Lambda_{\bar{u}}: Z_{T}^{0} \rightarrow Z_{T}^{0}$ is contractive let $\hat{u}_{1}, \hat{u}_{2} \in Z_{T}^{0}$. Then, (3.22) implies for $0 \leq t \leq T$,

$$
\begin{aligned}
\left\|\Lambda_{\bar{u}}\left[\hat{u}_{1}\right](t)-\Lambda_{\bar{u}}\left[\hat{u}_{2}\right](t)\right\|_{0} \leq & \int_{0}^{t}\left\|\mathbb{U}_{V_{\bar{u}}}(t, s)\right\|_{\mathcal{L}\left(E_{0}\right)}\left\|Q\left[\Lambda_{\bar{u}}\left[\hat{u}_{1}\right](s), \hat{u}_{1}(s)-\hat{u}_{2}(s)\right]\right\|_{0} \mathrm{~d} s \\
& +\int_{0}^{t}\left\|\mathbb{U}_{V_{\bar{u}}}(t, s)\right\|_{\mathcal{L}\left(E_{0}\right)}\left\|Q\left[\Lambda_{\bar{u}}\left[\hat{u}_{1}\right](s)-\Lambda_{\bar{u}}\left[\hat{u}_{2}\right](s), \hat{u}_{2}(s)\right]\right\|_{0} \mathrm{~d} s
\end{aligned}
$$

and hence Proposition 3.2, Lemma 3.1, and 3.24) give

$$
\begin{aligned}
\left\|\Lambda_{\bar{u}}\left[\hat{u}_{1}\right](t)-\Lambda_{\bar{u}}\left[\hat{u}_{2}\right](t)\right\|_{0} \leq & 2 S c_{*}\|\eta\|_{\infty} e^{\omega_{0}} T\left\|\hat{u}_{1}-\hat{u}_{2}\right\|_{C\left(J_{T}, E_{0}\right)} \\
& +2 S c_{*}\|\eta\|_{\infty} e^{\omega_{0}} \int_{0}^{t} \| \Lambda_{\bar{u}}\left[\hat{u}_{1}\right](s)-\Lambda_{\bar{u}}\left[\hat{u}_{2}(s) \|_{0} \mathrm{~d} s .\right.
\end{aligned}
$$

Gronwall's lemma implies

$$
\left\|\Lambda_{\bar{u}}\left[\hat{u}_{1}\right](t)-\Lambda_{\bar{u}}\left[\hat{u}_{2}\right](t)\right\|_{0} \leq T c(S)\left\|\hat{u}_{1}-\hat{u}_{2}\right\|_{C\left(J_{T}, E_{0}\right)}, \quad 0 \leq t \leq T .
$$

Consequently, for each $\bar{u} \in Z_{T}^{\nu}$ the mapping $\Lambda_{\bar{u}}$ defines a contraction on $Z_{T}^{0}$ when $T=T(S) \in(0,1]$ is chosen sufficiently small and $\Lambda_{\bar{u}}$ thus has a unique fixed point $\Gamma(\bar{u}) \in Z_{T}^{0}$. Recall that $\Gamma(\bar{u})$ belongs in addition to $C\left(J_{T}, E_{1}\right) \cap C^{1}\left(J_{T}, E_{0}\right)$ according to $(3.18)$.

We next study the mapping $\Gamma=[\bar{u} \mapsto \Gamma(\bar{u})]$ and show that it is a contraction on $Z_{T}^{\nu}$ provided $T=$ $T(S) \in(0,1]$ is sufficiently small. The corresponding unique fixed point along with the corresponding solution to (1.1) will then represent the local solution to (1.1)-(1.4). To this end note that (3.22) reads for the fixed point $u=\Gamma(\bar{u})$ of $\Lambda_{\bar{u}}$ (omitting the hat of $u$ for simplicity) as

$$
u(t)=\mathbb{U}_{V_{\bar{u}}}(t, 0) u^{0}+\int_{0}^{t} \mathbb{U}_{V_{\bar{u}}}(t, s) Q[u(s), u(s)] \mathrm{d} s, \quad t \in J_{T} .
$$

Now, consider $\bar{u}_{1}, \bar{u}_{2} \in Z_{T}^{\nu}$ and put $u_{1}:=\Gamma\left(\bar{u}_{1}\right)$ and $u_{2}:=\Gamma\left(\bar{u}_{2}\right)$. Then we infer from (3.26) for $t \in J_{T}$

$$
\begin{aligned}
\left\|u_{1}(t)-u_{2}(t)\right\|_{0} \leq & \left\|\mathbb{U}_{V_{\bar{u}_{1}}}(t, 0)-\mathbb{U}_{V_{\bar{u}_{2}}}(t, 0)\right\|_{\mathcal{L}\left(E_{1}, E_{0}\right)}\left\|u^{0}\right\|_{E_{1}} \\
& +\int_{0}^{t}\left\|\mathbb{U}_{V_{\bar{u}_{1}}}(t, s)-\mathbb{U}_{V_{\bar{u}_{2}}}(t, s)\right\|_{\mathcal{L}\left(E_{1}, E_{0}\right)}\left\|Q\left[u_{1}(s), u_{1}(s)\right]\right\|_{1} \mathrm{~d} s \\
& +\int_{0}^{t}\left\|\mathbb{U}_{V_{\bar{u}_{2}}}(t, s)\right\|_{\mathcal{L}\left(E_{0}\right)}\left\|Q\left[u_{2}(s), u_{1}(s)-u_{2}(s)\right]\right\|_{0} \mathrm{~d} s \\
& +\int_{0}^{t}\left\|\mathbb{U}_{V_{\bar{u}_{2}}}(t, s)\right\|_{\mathcal{L}\left(E_{0}\right)}\left\|Q\left[u_{1}(s)-u_{2}(s), u_{1}(s)\right]\right\|_{0} \mathrm{~d} s
\end{aligned}
$$

and hence, from Proposition 3.2, Lemma 3.1 , and (3.25),

$$
\begin{aligned}
\left\|u_{1}(t)-u_{2}(t)\right\|_{0} \leq & \omega_{0} T\left\|V_{\bar{u}_{1}}-V_{\bar{u}_{2}}\right\|_{C\left(J_{T}\right)}\left\|u^{0}\right\|_{E_{1}} \\
& +2 S c_{*}\|\eta\|_{\infty} m(S) \omega_{0} T\left\|V_{\bar{u}_{1}}-V_{\bar{u}_{2}}\right\|_{C\left(J_{T}\right)} \\
& +4 S c_{*}\|\eta\|_{\infty} e^{\omega_{0}} \int_{0}^{t}\left\|u_{1}(s)-u_{2}(s)\right\|_{0} \mathrm{~d} s .
\end{aligned}
$$

Gronwall's lemma implies

$$
\left\|u_{1}(t)-u_{2}(t)\right\|_{0} \leq T c(S)\left\|V_{\bar{u}_{1}}-V_{\bar{u}_{2}}\right\|_{C\left(J_{T}\right)}, \quad 0 \leq t \leq T,
$$


and thus, from $\underline{3.15}$,

$$
\left\|u_{1}(t)-u_{2}(t)\right\|_{0} \leq T c(S)\left\|\bar{u}_{1}-\bar{u}_{2}\right\|_{C\left(J_{T}, E_{0}\right)}, \quad 0 \leq t \leq T,
$$

for some constant $c(S)>0$. Next, (3.26) for $u=\Gamma(\bar{u})$ with $\bar{u} \in Z_{T}^{\nu}$ can also be written (see also (3.21)) as

$$
u^{\prime}+V_{\bar{u}}(t) \partial_{y}(\tau u)=Q[u(t), u(t)]+L[u(t)], \quad 0 \leq t \leq T .
$$

We shall integrate this equation with respect to $y \in\left(y_{0}, \infty\right)$. Note that $u(t) \in E_{1}$ and the assumption (2.10) on $\tau$ imply

$$
\int_{y_{0}}^{\infty} y \partial_{y}(\tau(y) u(t, y)) \mathrm{d} y=-\int_{y_{0}}^{\infty} \tau(y) u(t, y) \mathrm{d} y .
$$

Next, (2.3), 2.6), and (2.7) entail that

$$
\int_{y_{0}}^{\infty} y L[u(t)](y) \mathrm{d} y+\int_{y_{0}}^{\infty} y Q[u(t), u(t)](y) \mathrm{d} y=-\int_{y_{0}}^{\infty} y \mu(y) u(t, y) \mathrm{d} y-g(u(t)) .
$$

Consequently, we derive from (3.28)-(3.30) that

$$
\frac{\mathrm{d}}{\mathrm{d} t} \int_{y_{0}}^{\infty} y u(t, y) \mathrm{d} y=V_{\bar{u}}(t) \int_{y_{0}}^{\infty} \tau(y) u(t, y) \mathrm{d} y-\int_{y_{0}}^{\infty} y \mu(y) u(t, y) \mathrm{d} y-g(u(t)),
$$

for $0 \leq t \leq T$ and $u=\Gamma(\bar{u})$ with $\bar{u} \in Z_{T}^{\nu}$. In particular, (3.31) warrants

$$
\left|\frac{\mathrm{d}}{\mathrm{d} t} \nu\|u(t)\|_{0}\right| \leq \nu\left(V_{\bar{u}}(t) \frac{\|\tau\|_{\infty}}{y_{0}}+\|\mu\|_{\infty}+\|\beta\|_{\infty}\right)\|u(t)\|_{0} \leq r(S), \quad t \in J_{T},
$$

since (3.9), 3.12), and (3.14) imply

$$
V_{\bar{u}}(t) \leq v_{\bar{u}}(t) \leq S+2 S\|\beta\|_{\infty}+\lambda, \quad t \in J_{T},
$$

and hence $u=\Gamma(\bar{u}) \in Z_{T}^{\nu}$ for $\bar{u} \in Z_{T}^{\nu}$. Now, consider again $\bar{u}_{1}, \bar{u}_{2} \in Z_{T}^{\nu}$ and put $u_{1}:=\Gamma\left(\bar{u}_{1}\right)$ and $u_{2}:=\Gamma\left(\bar{u}_{2}\right)$. We then deduce from (3.31)

$$
\begin{aligned}
\left|\frac{\mathrm{d}}{\mathrm{d} t} \int_{y_{0}}^{\infty} y\left(u_{1}(t, y)-u_{2}(t, y)\right) \mathrm{d} y\right| \leq & \left|V_{\bar{u}_{1}}(t)-V_{\bar{u}_{2}}(t)\right| \int_{y_{0}}^{\infty} \tau(y) u_{1}(t, y) \mathrm{d} y \\
& +V_{\bar{u}_{2}}(t)\left|\int_{y_{0}}^{\infty} \tau(y)\left(u_{1}(t, y)-u_{2}(t, y)\right) \mathrm{d} y\right| \\
& +\left|\int_{y_{0}}^{\infty} y \mu(y)\left(u_{1}(t, y)-u_{2}(t, y)\right) \mathrm{d} y\right|+\left|g\left(u_{1}(t)-u_{2}(t)\right)\right| .
\end{aligned}
$$

Since the kernels are bounded we obtain

$$
\begin{aligned}
\left|\frac{\mathrm{d}}{\mathrm{d} t} \int_{y_{0}}^{\infty} y\left(u_{1}(t, y)-u_{2}(t, y)\right) \mathrm{d} y\right| \leq & c(S)\left|V_{\bar{u}_{1}}(t)-V_{\bar{u}_{2}}(t)\right|+c(S)\left\|u_{1}(t)-u_{2}(t)\right\|_{0} \\
& +\left|g\left(u_{1}(t)-u_{2}(t)\right)\right| .
\end{aligned}
$$

Invoking (3.14), 3.16), and (3.27) we get

$$
\left|\frac{\mathrm{d}}{\mathrm{d} t} \int_{y_{0}}^{\infty} y\left(u_{1}(t, y)-u_{2}(t, y)\right) \mathrm{d} y\right| \leq T c(S) d_{Z_{T}^{\nu}}\left(\bar{u}_{1}, \bar{u}_{2}\right), \quad 0 \leq t \leq T .
$$

Combining (3.27) and (3.32) shows that

$$
d_{Z_{T}^{\nu}}\left(\Gamma\left(\bar{u}_{1}\right), \Gamma\left(\bar{u}_{2}\right)\right) \leq T c(S) d_{Z_{T}^{\nu}}\left(\bar{u}_{1}, \bar{u}_{2}\right), \quad \bar{u}_{1}, \bar{u}_{2} \in Z_{T}^{\nu},
$$

that is, $\bar{u} \mapsto \Gamma(\bar{u})$ is a contraction on $Z_{T}^{\nu}$ provided that $T=T(S) \in(0,1]$ is chosen sufficiently small. The contraction mapping principle then yields a unique fixed point $u$ so that $\left(v_{u}, u\right)$ is the unique solution to (1.1)-(1.4) on the interval [0,T]. Since the choice of $T=T(S)$ only depends on $S$ from (3.9), the following statement is immediate: 
Proposition 3.3. Given the assumptions of Theorem 2.1] there exists a unique maximal solution $(v, u)$ to (1.1)-(1.4) belonging to $C\left(J, \mathbb{R}^{+} \times E_{1}^{+}\right) \cap C^{1}\left(J, \mathbb{R} \times E_{0}\right)$ on a maximal interval $J$ which is open in $\mathbb{R}^{+}$. If $t^{+}:=\sup J<\infty$, then

$$
\liminf _{t \nearrow t^{+}} v(t)=0 \quad \text { or } \quad \limsup _{t \nearrow t^{+}}\left(v(t)+\|u(t)\|_{E_{1}}\right)=\infty .
$$

Let us point out that the solution $(v, u)$ satisfies

$$
u^{\prime}+\mathbb{A}_{V_{u}}(t) u=Q[u, u], \quad t \in J, \quad u(0)=u^{0},
$$

with $V_{u}$ being defined in (3.13) and $u$ can thus be written as

$$
u(t)=\mathbb{U}_{V_{u}}(t, 0) u^{0}+\int_{0}^{t} \mathbb{U}_{V_{u}}(t, s) Q[u(s), u(s)] \mathrm{d} s, \quad t \in J .
$$

3.3. Global Existence. We next show that (3.33) cannot occur and the solution provided by Proposition 3.3 thus exists on $J=\mathbb{R}^{+}$. For this we note the monomer balance law

$$
\dot{v}(t)+\frac{\mathrm{d}}{\mathrm{d} t} \int_{y_{0}}^{\infty} y u(t, y) \mathrm{d} y=\lambda-\gamma v(t)-\int_{y_{0}}^{\infty} y \mu(y) u(t, y) \mathrm{d} y, \quad t \in J,
$$

which now readily follows from (3.31) and (1.1). This turns out to be crucial for global existence as it implies the a priori bound

$$
v(t)+\|u(t)\|_{E_{0}} \leq v^{0}+\left\|u^{0}\right\|_{E_{0}}+\lambda t, \quad t \in J .
$$

We then argue by contradiction and suppose that $t^{+}<\infty$. Recalling (1.1) we derive from (3.14) and (3.35)

$$
v^{\prime}(t) \leq \lambda+g(u(t)) \leq \lambda+\|\beta\|_{\infty}\|u(t)\|_{E_{0}} \leq c\left(t^{+}\right),
$$

while (3.35) also implies

$$
v^{\prime}(t) \geq-\gamma v(t)-\frac{v(t)}{1+\nu\|u(t)\|_{0}} \int_{y_{0}}^{\infty} \tau(y) u(t, y) \mathrm{d} y \geq-\gamma v(t)-v(t)\|\tau\|_{\infty}\|u(t)\|_{0} \geq-c\left(t^{+}\right)
$$

for $t \in J$. Therefore,

$$
\|v\|_{C^{1}(J)} \leq c\left(t^{+}\right) .
$$

Furthermore, (3.12) and (3.14) along with (3.35) warrant

$$
v(t) \geq \exp \left(-\gamma t-\int_{0}^{t} p(\bar{u}(\sigma)) \mathrm{d} \sigma\right) v^{0} \geq \exp \left(-\gamma t^{+}-c\left(t^{+}\right)\right) v^{0}>0
$$

for $t \in J$. Consequently, there exists $R=R\left(c\left(t^{+}\right)\right)>0$ such that for each $0<T<t^{+}$we have $v \in \mathcal{V}_{T, R}$. Hence, it follows from Proposition 3.2 that

$$
\left\|\mathbb{U}_{V_{u}}(t, s)\right\|_{\mathcal{L}\left(E_{1}\right)} \leq c\left(t^{+}\right), \quad 0 \leq s \leq t<t^{+} .
$$

We next infer from Lemma 3.1 and (3.35) that

$$
\|Q[u(t), u(t)]\|_{1} \leq c_{*}\|\eta\|_{\infty}\|u(t)\|_{1}\|u(t)\|_{0} \leq c\left(t^{+}\right)\|u(t)\|_{1}, \quad t \in J .
$$

Therefore,

$$
\begin{aligned}
\|u(t)\|_{1} & \leq\left\|\mathbb{U}_{V_{u}}(t, 0)\right\|_{\mathcal{L}\left(E_{1}\right)}\left\|u^{0}\right\|_{1}+\int_{0}^{t}\left\|\mathbb{U}_{V_{u}}(t, s)\right\|_{\mathcal{L}\left(E_{1}\right)}\|Q[u(s), u(s)]\|_{1} \mathrm{~d} s \\
& \leq c\left(t^{+}\right)\left\|u^{0}\right\|_{1}+c\left(t^{+}\right) \int_{0}^{t}\|u(s)\|_{1} \mathrm{~d} s, \quad t \in J
\end{aligned}
$$

so that Gronwall's lemma ensures

$$
\|u(t)\|_{1} \leq c\left(t^{+}\right), \quad t \in J .
$$

Consequently, (3.36), (3.37), and (3.39) rule out the occurrence of (3.33) contradicting our assumption of a finite $t^{+}$, hence $t^{+}=\infty$. This completes the proof of Theorem 2.1. 
3.4. Finite Speed of Propagation. For later purposes when dealing with weak solutions we consider compactly supported initial values and show that the support propagates with finite speed provided that large polymers do not join.

Lemma 3.4. Let the assumptions of Theorem 2.1] hold and suppose there is $S_{1}>0$ such that $\eta(y, z)=0$ for $(y, z) \in Y \times Y$ with $z+y>S_{1}$. Let $v^{0}>0$ and $u^{0} \in E_{1} \cap E_{0}^{+}$with $\operatorname{supp} u^{0} \subset\left[y_{0}, S_{0}\right]$ for some $S_{0}>y_{0}$. Let $(v, u)$ be the corresponding solution to (1.1)-(1.4) provided by Theorem [2.1] Then

$$
\operatorname{supp} u(t) \subset\left[y_{0}, S(t)\right], \quad t \geq 0,
$$

where $S$ is the solution to the ode

$$
S^{\prime}(t)=\frac{v(t)}{1+\nu\|u(t)\|_{E_{0}}} \tau(S), \quad t>0, \quad S(0)=\max \left\{S_{0}, S_{1}\right\}
$$

that is,

$$
S(t)=\phi^{-1}\left(\int_{0}^{t} \frac{v(s)}{1+\nu\|u(s)\|_{E_{0}}} \mathrm{~d} s\right) \quad \text { with } \quad \phi(r):=\int_{\max \left\{S_{0}, S_{1}\right\}}^{r} \frac{\mathrm{d} z}{\tau(z)} .
$$

Proof. The proof follows along the lines of [19, Lemma 2.4]. Indeed, noticing that $S$ is well-defined on $\mathbb{R}^{+}$, since $\tau$ is a bounded and continuous function, and defining $P \in C^{1}\left(\mathbb{R}^{+}, L_{1}(Y)\right)$ according to

$$
P(t, y):=\int_{y}^{\infty} u\left(t, y^{\prime}\right) \mathrm{d} y^{\prime}, \quad y \in Y, t \geq 0
$$

we note that

$$
\begin{aligned}
\frac{\partial}{\partial t} P(t, y) & =\int_{y}^{\infty} \partial_{t} u\left(t, y^{\prime}\right) \mathrm{d} y^{\prime} \\
& =\frac{v(t)}{1+\nu\|u(t)\|_{E_{0}}} \tau(y) u(t, y)+\int_{y}^{\infty} L[u(t)]\left(y^{\prime}\right) \mathrm{d} y^{\prime}+\int_{y}^{\infty} Q[u(t), u(t)]\left(y^{\prime}\right) \mathrm{d} y^{\prime}
\end{aligned}
$$

Since

$$
\begin{aligned}
\int_{S(t)}^{\infty} \int_{y}^{\infty} Q[u(t), u(t)]\left(y^{\prime}\right) \mathrm{d} y^{\prime} \mathrm{d} y= & \int_{S(t)}^{\infty} \int_{y \vee 2 y_{0}}^{\infty} \int_{y_{0}}^{y^{\prime}-y_{0}} \eta\left(y^{\prime}-z, z\right) u\left(t, y^{\prime}-z\right) u(t, z) \mathrm{d} z \mathrm{~d} y^{\prime} \mathrm{d} y \\
& -2 \int_{S(t)}^{\infty} \int_{y}^{\infty} u\left(t, y^{\prime}\right) \int_{y_{0}}^{\infty} \eta\left(z, y^{\prime}\right) u(t, z) \mathrm{d} z \mathrm{~d} y^{\prime} \mathrm{d} y,
\end{aligned}
$$

we obtain from the assumption on $\eta$ that

$$
\int_{S(t)}^{\infty} \int_{y}^{\infty} Q[u(t), u(t)]\left(y^{\prime}\right) \mathrm{d} y^{\prime} \mathrm{d} y=0, \quad t \geq 0
$$


Therefore, using the positivity of $u$, (2.4) (which is implied by (2.2), (2.3)), and the definition of $S$, we compute

$$
\begin{aligned}
\frac{\mathrm{d}}{\mathrm{d} t} \int_{S(t)}^{\infty} P(t, y) \mathrm{d} y= & \int_{S(t)}^{\infty} \frac{\partial}{\partial t} P(t, y) \mathrm{d} y-S^{\prime}(t) P(t, S(t)) \\
= & \frac{-v(t)}{1+\nu\|u(t)\|_{E_{0}}} \int_{S(t)}^{\infty} \tau(y) \partial_{y} P(t, y) \mathrm{d} y+\int_{S(t)}^{\infty} \int_{y}^{\infty} L[u(t)]\left(y^{\prime}\right) \mathrm{d} y^{\prime} \mathrm{d} y \\
& -\frac{v(t)}{1+\nu\|u(t)\|_{E_{0}}} \tau(S(t)) P(t, S(t)) \\
= & \frac{v(t)}{1+\nu\|u(t)\|_{E_{0}}} \int_{S(t)}^{\infty} \tau^{\prime}(y) P(t, y) \mathrm{d} y+\int_{S(t)}^{\infty} \int_{y}^{\infty} L[u(t)]\left(y^{\prime}\right) \mathrm{d} y^{\prime} \mathrm{d} y \\
\leq & \left\|\tau^{\prime}\right\|_{\infty} v(t) \int_{S(t)}^{\infty} P(t, y) \mathrm{d} y+2 \int_{S(t)}^{\infty} \int_{y}^{\infty} \beta\left(y^{\prime \prime}\right) u\left(t, y^{\prime \prime}\right) \int_{y}^{y^{\prime \prime}} \kappa\left(y^{\prime}, y^{\prime \prime}\right) \mathrm{d} y^{\prime} \mathrm{d} y^{\prime \prime} \mathrm{d} y \\
\leq & \left\|\tau^{\prime}\right\|_{\infty} v(t) \int_{S(t)}^{\infty} P(t, y) \mathrm{d} y+2\|\beta\|_{\infty} \int_{S(t)}^{\infty} P(t, y) \mathrm{d} y .
\end{aligned}
$$

Thus, Gronwall's lemma along with

$$
\int_{S(0)}^{\infty} P(0, y) \mathrm{d} y=0
$$

implies

$$
\int_{S(t)}^{\infty} P(t, y) \mathrm{d} y=0, \quad t \geq 0
$$

guaranteeing that $u(t, \cdot)$ vanishes on the interval $(S(t), \infty)$ for each $t \geq 0$.

\section{Proof of Theorem 2.3}

We shall prove Theorem 2.3 for unbounded kernels and thus suppose the conditions sated therein. Recall that $v^{0}>0$ and $u^{0} \in L_{1}^{+}(Y, y \mathrm{~d} y)$. We fix an arbitrary $T>0$.

We first construct a suitable bounded approximation of the unbounded kernels for which classical solutions exist according to Theorem 2.1 and we show then that a cluster point exists that is a weak solution for the original unbounded kernels. This approach follows along the lines of [13] but requires extensions particularly due to the polymer joining term. For this we borrow ideas from [6] (see also [11]) used on the coagulation-fragmentation equations.

4.1. Approximation by Bounded Kernels. Let us first observe that $u^{0} \in L_{1}^{+}(Y, y \mathrm{~d} y)$ implies that we can apply a refined version of the de la Vallée-Poussin Theorem [10] guaranteeing the existence of non-negative, non-decreasing, and convex function $\Phi \in C^{\infty}\left(\mathbb{R}^{+}\right)$with $\Phi(0)=0$ such that $\Phi^{\prime}$ is concave and

$$
\lim _{r \rightarrow \infty} \Phi^{\prime}(r)=\lim _{r \rightarrow \infty} \frac{\Phi(r)}{r}=\infty
$$

with

$$
\int_{y_{0}}^{\infty} \Phi(y) u^{0}(y) \mathrm{d} y<\infty .
$$

We may then choose a sequence $\left(u_{n}^{0}\right)_{n \in \mathbb{N}}$ of non-negative, smooth, and compactly supported functions such that

$$
u_{n}^{0} \rightarrow u^{0} \quad \text { in } \quad L_{1}^{+}(Y) \quad \text { and } \quad \sup _{n \in \mathbb{N}} \int_{y_{0}}^{\infty} \Phi(y) u_{n}^{0}(y) \mathrm{d} y<\infty .
$$

Next, we use a mollifier argument to construct a sequence $\left(\tau_{n}\right)_{n \in \mathbb{N}}$ in $B U C^{\infty}\left(\left[y_{0}, \infty\right)\right)$ satisfying

$$
0<\frac{\tau_{0}}{2} \leq \tau_{n}(y) \leq \tau_{*} y, \quad y \geq y_{0},
$$


such that

$$
\tau_{n} \rightarrow \tau \quad \text { uniformly on compact subsets of } Y .
$$

Moreover, we can choose a sequence $\left(\eta_{n}\right)_{n \in \mathbb{N}}$ in $B U C^{\infty}(Y \times Y)$ such that

$$
\eta_{n}(y, z)=\eta_{n}(z, y) \leq K\left(y^{\alpha} z^{\rho}+y^{\rho} z^{\alpha}\right), \quad y, z \in Y,
$$

with constants $K, \alpha$, and $\rho$ stemming from 2.17) and

$$
\eta_{n}(y, z)=0 \text { for }(y, z) \text { with } y+z>R_{n}, \quad 2 y_{0}<R_{n} \rightarrow \infty,
$$

and such that

$$
\eta_{n} \rightarrow \eta \quad \text { uniformly on compact subsets of } Y \times Y \text {. }
$$

For $n \in \mathbb{N}$ we put

$$
S_{n}^{0}:=\sup \left\{y \in\left(y_{0}, \infty\right): y \in \operatorname{supp} u_{n}^{0}\right\}
$$

and

$$
H_{n}(T):=\phi_{n}^{-1}\left(\int_{0}^{T}\left(v_{0}+\int_{y_{0}}^{\infty} y u_{n}^{0}(y) \mathrm{d} y+\lambda t\right) \mathrm{d} t\right), \quad \phi_{n}(r):=\int_{\max \left\{S_{n}^{0}, R_{n}\right\}}^{r} \frac{\mathrm{d} z}{\tau_{n}(z)}
$$

and then introduce

$$
\mathcal{S}_{n}(T):=\max \left\{\mathcal{S}_{n-1}(T), H_{n}(T), n\right\}, \quad n \geq 1, \quad \mathcal{S}_{0}(T):=H_{0}(T) .
$$

Let $\mu_{n}:=\mathbf{1}_{\left[y_{0}, \mathcal{S}_{n}(T)\right]} \mu$ and $\beta_{n}:=\mathbf{1}_{\left[y_{0}, \mathcal{S}_{n}(T)\right]} \beta$ for $n \in \mathbb{N}$. Thus, Theorem 2.1 ensures the existence of a global non-negative classical solution

$$
\left(v_{n}, u_{n}\right) \in C^{1}\left(\mathbb{R}^{+}, \mathbb{R} \times E_{0}\right) \cap C\left(\mathbb{R}^{+}, \mathbb{R} \times E_{1}\right)
$$

to (1.1)-1.2 when $\left(\tau, \mu, \beta, \eta, u^{0}\right)$ is replaced with $\left(\tau_{n}, \mu_{n}, \beta_{n}, \eta_{n}, u_{n}^{0}\right)$. Moreover, the construction of $\mathcal{S}_{n}$ together with Lemma 3.4 (3.35), and $\beta>0$ imply

$$
\operatorname{supp} u_{n}(t) \subset\left[y_{0}, \mathcal{S}_{n}(T)\right]=\operatorname{supp} \beta_{n}, \quad t \in[0, T] .
$$

From (2.8) and (4.2) we have

$$
v_{n}(t)+\int_{y_{0}}^{\infty} y u_{n}(t, y) \mathrm{d} y+\int_{0}^{t} \int_{y_{0}}^{\infty} y \mu_{n}(y) u_{n}(s, y) \mathrm{d} y \mathrm{~d} s \leq c(T), \quad t \in[0, T], \quad n \in \mathbb{N},
$$

where $c(T)$ is independent of $n$. We shall use in the following the notation

$$
L_{n}[u](y):=-\left(\mu_{n}(y)+\beta_{n}(y)\right) u(y)+2 \int_{y}^{\infty} \beta_{n}(z) \kappa(y, z) u(z) \mathrm{d} z, \quad y \in Y,
$$

and

$$
Q_{n}[u](y):=\mathbf{1}_{\left[y>2 y_{0}\right]} \int_{y_{0}}^{y-y_{0}} \eta_{n}(y-z, z) u(y-z) u(z) \mathrm{d} z-2 u(y) \int_{y_{0}}^{\infty} \eta_{n}(z, y) u(z) \mathrm{d} z, \quad y \in Y .
$$

In order to deal with the bilinear polymer joining terms we adapt the ideas from [6, Lemma 3.2] (on the coagulation-fragmentation equations) to our situation and derive some estimates on the moments

$$
M_{s, n}(t):=\int_{y_{0}}^{\infty} y^{s} u_{n}(t, y) \mathrm{d} y, \quad t \in[0, T],
$$

for $s>0$ and $n \in \mathbb{N}$. Note that all moments are well-defined due to the compact support of $u_{n}(t, \cdot)$.

Lemma 4.1. Let $\theta=\alpha+\rho \in(1,2]$ in 2.17) and recall that then 2.19) is supposed to hold. There is a constant $C_{M}(T)$ independent of $n$ such that

$$
M_{2, n}(t) \leq C_{M}(T)\left(1+t^{-1 / \zeta}\right), \quad t \in[0, T], \quad n \in \mathbb{N} .
$$


Proof. As pointed out the proof follows along the lines of [6, Lemma 3.2]. Note that owing to (4.5) we have

$$
\eta_{n}(y, z) \leq 2 K\left(y^{\theta}+z^{\theta}\right), \quad(y, z) \in Y \times Y
$$

and so it follows form (2.7) and (4.9) for $t \in[0, T]$

$$
\begin{aligned}
\int_{y_{0}}^{\infty} & y^{2} Q_{n}\left[u_{n}(t)\right](y) \mathrm{d} y \\
& \leq 2 K \int_{y_{0}}^{\infty} \int_{y_{0}}^{\infty}\left(y^{\theta}+z^{\theta}\right)\left((y+z)^{2}-y^{2}-z^{2}\right) u_{n}(t, y) u_{n}(t, z) \mathrm{d} z \mathrm{~d} y \\
& =8 K \int_{y_{0}}^{\infty} \int_{y_{0}}^{\infty} y^{1+\theta} z u_{n}(t, y) u_{n}(t, z) \mathrm{d} z \mathrm{~d} y
\end{aligned}
$$

hence

$$
\int_{y_{0}}^{\infty} y^{2} Q_{n}\left[u_{n}(t)\right](y) \mathrm{d} y \leq c(T) M_{1+\theta, n}(t) .
$$

In addition, (2.6), (2.19), (4.8), and the positivity of $\mu_{n}, \beta_{n}$ and $u_{n}$ imply for $t \in[0, T]$

$$
\begin{aligned}
-\int_{y_{0}}^{\infty} y^{2} L_{n}\left[u_{n}(t)\right](y) \mathrm{d} y= & \int_{y_{0}}^{\infty} y^{2}\left(\mu_{n}(y)+\beta_{n}(y)\right) u_{n}(t, y) \mathrm{d} y \\
& \quad-2 \int_{y_{0}}^{\infty} \beta_{n}(y) u_{n}(t, y) \int_{y_{0}}^{y} z^{2} \kappa(z, y) \mathrm{d} z \mathrm{~d} y \\
\geq & (1-a) B \int_{y_{0}}^{\infty} y^{2+\zeta} u_{n}(t, y) \mathrm{d} y \\
& =(1-a) B M_{2+\zeta, n}(t) .
\end{aligned}
$$

Next, using integration by parts we obtain from 2.14

$$
\int_{y_{0}}^{\infty} y^{2} \partial_{y}\left(\tau_{n}(y) u_{n}(t, y)\right) \mathrm{d} y=\left.y^{2}\left(\tau_{n}(y) u_{n}(t, y)\right)\right|_{y_{0}} ^{\infty}-2 \int_{y_{0}}^{\infty} y \tau_{n}(y) u_{n}(t, y) \mathrm{d} y \geq-2 \tau_{*} M_{2, n}(t) .
$$

Therefore, integrating (1.2) with respect to $y^{2} \mathrm{~d} y$ and using the above estimates and (4.9) we deduce

$$
\begin{aligned}
\frac{\mathrm{d} M_{2, n}(t)}{\mathrm{d} t}= & -\frac{v_{n}(t)}{1+\nu\left\|u_{n}(t)\right\|_{0}} \int_{y_{0}}^{\infty} y^{2} \partial_{y}\left(\tau_{n}(y) u_{n}(t, y)\right) \mathrm{d} y \\
& +\int_{y_{0}}^{\infty} y^{2} L_{n}\left[u_{n}(t)\right](y) \mathrm{d} y+\int_{y_{0}}^{\infty} y^{2} Q_{n}\left[u_{n}(t)\right](y) \mathrm{d} y \\
\leq & c(T) M_{2, n}(t)-(1-a) B M_{2+\zeta, n}(t)+c(T) M_{1+\theta, n}(t),
\end{aligned}
$$

hence, since $1+\theta>2$,

$$
\frac{\mathrm{d} M_{2, n}(t)}{\mathrm{d} t}+c_{1} M_{2+\zeta, n}(t) \leq c(T) M_{1+\theta, n}(t), \quad t \in[0, T] .
$$

Next, since $\zeta>\theta-1$, Hölder's inequality and the fact that $M_{1, n}(t) \leq c(T)$ by (4.9) imply

$$
M_{1+\theta, n}(t) \leq M_{1, n}^{\frac{1+\zeta-\theta}{1+\zeta}}(t) M_{2+\zeta, n}^{\frac{\theta}{1+\zeta}}(t) \leq c(T) M_{2+\zeta, n}^{\frac{\theta}{1+\zeta}}(t)
$$

and plugging this into 4.11) and using Young's inequality (noticing that $\theta<1+\zeta$ ) we derive

$$
\frac{\mathrm{d} M_{2, n}(t)}{\mathrm{d} t}+c_{1}(T) M_{2+\zeta, n}(t) \leq c(T), \quad t \in[0, T] .
$$

Finally, using again Hölder's inequality and (4.9) we get

$$
M_{2, n}(t) \leq M_{1, n}^{\frac{\zeta}{1+\zeta}}(t) M_{2+\zeta, n}^{\frac{1}{1+\zeta}}(t) \leq c(T) M_{2+\zeta, n}^{\frac{1}{1+\zeta}}(t)
$$


and so

$$
\frac{\mathrm{d} M_{2, n}(t)}{\mathrm{d} t}+c_{1}(T) M_{2, n}^{1+\zeta}(t) \leq c(T), \quad t \in[0, T]
$$

The fact that the corresponding differential equation is solved by $t \mapsto C_{M}\left(1+t^{-\frac{1}{\zeta}}\right)$ with $C_{M}$ only depending on $K, B, \theta, \zeta$, and $T$ yields the assertion.

Corollary 4.2. Let $\theta=\alpha+\rho \in(1,2]$ in 2.17) and assume 2.19). Then

$$
\int_{0}^{T} M_{\theta, n}(t) \mathrm{d} t \leq c(T), \quad t \in[0, T], \quad n \in \mathbb{N},
$$

with a constant $c(T)$ not depending on $n \in \mathbb{N}$.

Proof. Noticing that Hölder's inequality, 4.9, and Lemma 4.1 imply

$$
M_{\theta, n}(t) \leq M_{1, n}(t)^{2-\theta} M_{2, n}(t)^{\theta-1} \leq c(T)\left(1+t^{-\frac{1}{\zeta}}\right)^{\theta-1} \leq c(T)\left(1+t^{-\frac{\theta-1}{\zeta}}\right)
$$

for $t \in[0, T]$, the assertion follows since $\theta-1<\zeta$.

We next derive a priori estimates which imply then later on the compactness of the sequence $\left(\left(v_{n}, u_{n}\right)\right)_{n \in \mathbb{N}}$.

Lemma 4.3. There exists a constant $c(T)$ independent of $n$ such that

$$
\begin{gathered}
\int_{y_{0}}^{\infty} \Phi(y) u_{n}(t, y) \mathrm{d} y \leq c(T), \\
\int_{0}^{t} I_{1, n}(s) \mathrm{d} s+\int_{0}^{t} I_{2, n}(s) \mathrm{d} s \leq c(T), \\
\int_{0}^{t} \int_{y_{0}}^{\infty} \Phi(y) \mu_{n}(y) u_{n}(s, y) \mathrm{d} y \mathrm{~d} s \leq c(T),
\end{gathered}
$$

for $t \in[0, T]$, where

$$
\begin{gathered}
I_{1, n}(s):=\int_{y_{0}}^{\infty} u_{n}(s, y) \beta_{n}(y) \int_{y_{0}}^{y}\left(\frac{\Phi(y)}{y}-\frac{\Phi(z)}{z}\right) z \kappa(z, y) \mathrm{d} z \mathrm{~d} y \\
I_{2, n}(s):=\int_{y_{0}}^{\infty} u_{n}(s, y) \beta_{n}(y) \frac{\Phi(y)}{y} \int_{0}^{y_{0}} z \kappa(z, y) \mathrm{d} z \mathrm{~d} y
\end{gathered}
$$

Proof. Recalling that $u_{n}(t, \cdot)$ is compactly supported we may test the corresponding equation (1.2) with $\Phi$ and obtain for $t \in[0, T]$ and $n \in \mathbb{N}$ on using (2.6) and (2.7) that

$$
\begin{aligned}
\int_{y_{0}}^{\infty} \Phi(y) u_{n}(t, y) \mathrm{d} y= & \int_{y_{0}}^{\infty} \Phi(y) u_{n}^{0}(y) \mathrm{d} y+\int_{0}^{t} \frac{v_{n}(s)}{1+\nu\left\|u_{n}(s)\right\|_{0}} \int_{y_{0}}^{\infty} \Phi^{\prime}(y) \tau_{n}(y) u_{n}(s, y) \mathrm{d} y \mathrm{~d} s \\
& +\int_{0}^{t} \int_{y_{0}}^{\infty} \int_{y_{0}}^{\infty} \tilde{\Phi}(y, z) \eta_{n}(y, z) u_{n}(y) u_{n}(z) \mathrm{d} z \mathrm{~d} y \mathrm{~d} s \\
& -\int_{0}^{t} \int_{y_{0}}^{\infty} \Phi(y)\left(\mu_{n}(y)+\beta_{n}(y)\right) u_{n}(s, y) \mathrm{d} y \mathrm{~d} s \\
& +2 \int_{0}^{t} \int_{y_{0}}^{\infty} u_{n}(s, y) \beta_{n}(y) \int_{y_{0}}^{y} \Phi(z) \kappa(z, y) \mathrm{d} z \mathrm{~d} y \mathrm{~d} s
\end{aligned}
$$


where $\tilde{\Phi}(y, z):=\Phi(y+z)-\Phi(y)-\Phi(z)$ for $y, z \in Y$. We may rewrite the last two integrals on the right hand side using (2.3) to get

$$
\begin{aligned}
& \int_{y_{0}}^{\infty} \Phi(y) u_{n}(t, y) \mathrm{d} y=\int_{y_{0}}^{\infty} \Phi(y) u_{n}^{0}(y) \mathrm{d} y+\int_{0}^{t} \frac{v_{n}(s)}{1+\nu\left\|u_{n}(s)\right\|_{0}} \int_{y_{0}}^{\infty} \Phi^{\prime}(y) \tau_{n}(y) u_{n}(s, y) \mathrm{d} y \mathrm{~d} s \\
&-\int_{0}^{t} \int_{y_{0}}^{\infty} \Phi(y) \mu_{n}(y) u_{n}(s, y) \mathrm{d} y \mathrm{~d} s \\
&+\int_{0}^{t} \int_{y_{0}}^{\infty} \int_{y_{0}}^{\infty} \tilde{\Phi}(y, z) \eta_{n}(y, z) u_{n}(s, y) u_{n}(s, z) \mathrm{d} z \mathrm{~d} y \mathrm{~d} s \\
&-2 \int_{0}^{t}\left(I_{1, n}(s)+I_{2, n}(s)\right) \mathrm{d} s .
\end{aligned}
$$

We then argue as in [13, Section 4]. Clearly, the terms involving $\mu_{n}$ and $I_{2, n}(s)$ are non-negative. The convexity of $\Phi$ and $\Phi(0)=0$ imply that the mapping $y \mapsto \Phi(y) / y$ is non-decreasing so that $I_{1, n}(s)$ is non-negative as well. On the other hand, the convexity of $\Phi^{\prime}$ along with $\Phi^{\prime}(0) \geq 0$ entails

$$
-\Phi^{\prime}(y) \leq \Phi^{\prime}(0)-\Phi^{\prime}(y) \leq-y \Phi^{\prime \prime}(y)
$$

and integrating this inequality yields $y \Phi^{\prime}(y) \leq 2 \Phi(y)$ for $y \in Y$. Hence, since $\Phi^{\prime} \geq 0$, we obtain from (2.14) and (4.9)

$$
\begin{aligned}
\int_{0}^{t} \frac{v_{n}(s)}{1+\nu\left\|u_{n}(s)\right\|_{0}} \int_{y_{0}}^{\infty} \Phi^{\prime}(y) \tau_{n}(y) u_{n}(s, y) \mathrm{d} y \mathrm{~d} s & \leq c(T) \int_{0}^{t} \int_{y_{0}}^{\infty} y \Phi^{\prime}(y) u_{n}(s, y) \mathrm{d} y \mathrm{~d} s \\
& \leq c(T) \int_{0}^{t} \int_{y_{0}}^{\infty} \Phi(y) u_{n}(s, y) \mathrm{d} y \mathrm{~d} s .
\end{aligned}
$$

To bound the integral term involving $\eta_{n}$ in (4.15) we argue along the lines of [6, Proposition 3.4]. As therein we first note that (since $\Phi$ is convex and non-decreasing together with [12, Lemma A.2])

$$
0 \leq \tilde{\Phi}(y, z) \leq 2 \frac{z \Phi(y)+y \Phi(z)}{y+z}, \quad(y, z) \in Y \times Y,
$$

which shows that the integral term involving $\eta_{n}$ is non-negative. Introducing

$$
\Psi(y, z):=\tilde{\Phi}(y, z) y^{\alpha} z^{\rho}, \quad(y, z) \in Y \times Y,
$$

we also obtain from (4.17)

$$
\Psi(y, z) \leq 2(z \Phi(y)+y \Phi(z)),
$$

if $\theta=\alpha+\rho \leq 1$. If $\theta \in(1,2]$ and $y \geq z$, then (4.17) implies

$$
\Psi(y, z) \leq 2 \frac{y z^{\theta} \Phi(y)+y y^{\theta} \Phi(z)}{y+z} \leq 2\left(z^{\theta} \Phi(y)+y^{\theta} \Phi(z)\right)
$$

while the case $y \leq z$ is analogous. We set $\theta_{1}:=\max \{1, \theta\}$ and obtain from (4.5) and the estimates on $\Psi$

$$
\begin{aligned}
\int_{0}^{t} \int_{y_{0}}^{\infty} & \int_{y_{0}}^{\infty} \tilde{\Phi}(y, z) \eta_{n}(y, z) u_{n}(s, y) u_{n}(s, z) \mathrm{d} z \mathrm{~d} y \mathrm{~d} s \\
& \leq 2 K \int_{0}^{t} \int_{y_{0}}^{\infty} \int_{y_{0}}^{\infty} \Psi(y, z) u_{n}(s, y) u_{n}(s, z) \mathrm{d} z \mathrm{~d} y \mathrm{~d} s \\
& \leq 8 K \int_{0}^{t} M_{\theta_{1}, n}(s) \int_{y_{0}}^{\infty} \Phi(y) u_{n}(s, y) \mathrm{d} y \mathrm{~d} s .
\end{aligned}
$$


From (4.9) and Corollary 4.2 we know that $\int_{0}^{T} M_{\theta_{1}, n}(s) \mathrm{d} s$ is bounded independent of $n$. Therefore, the estimates (4.2), (4.16), and (4.18) allow us to apply Gronwall's inequality to (4.15) in order to deduce that

$$
\int_{y_{0}}^{\infty} \Phi(y) u_{n}(t, y) \mathrm{d} y+\int_{0}^{t} I_{1, n}(s) \mathrm{d} s+\int_{0}^{t} I_{2, n}(s) \mathrm{d} s+\int_{0}^{t} \int_{y_{0}}^{\infty} \Phi(y) \mu_{n}(y) u_{n}(s, y) \mathrm{d} y \mathrm{~d} s \leq c(T),
$$

whence the claim.

4.2. Compactness. The estimates stated in Lemma 4.3 allow us to show the weak compactness of the sequence $\left(\left(v_{n}, u_{n}\right)\right)_{n \in \mathbb{N}}$ following [6, 13].

Proposition 4.4. There is a weakly compact subset $K_{T}$ of $L_{1}(Y, y \mathrm{~d} y)$ such that $u_{n}(t) \in K_{T}$ for $n \in \mathbb{N}$ and $0 \leq t \leq T$. Moreover,

$$
\int_{0}^{T} \int_{y_{0}}^{\infty} \beta_{n}(y) u_{n}(s, y) \mathrm{d} y \mathrm{~d} s \leq c(T), \quad n \in \mathbb{N},
$$

for some positive constant $c(T)$ independent of $n \in \mathbb{N}$.

Proof. Given $n \in \mathbb{N}$ and $t \in[0, T]$ it follows exactly as in [13, Lemma 4.1] that the properties of $\Phi$ and (4.12) imply

$$
\lim _{R \rightarrow \infty} \sup _{\substack{n \in \mathbb{N} \\ t \in[0, T]}} \int_{R}^{\infty} u_{n}(t, y) y \mathrm{~d} y=0
$$

and, for $S>R>2 y_{0}$ fixed,

$$
\int_{S}^{\infty} u_{n}(s, y) \beta_{n}(y) \int_{y_{0}}^{R} \kappa(z, y) \mathrm{d} z \mathrm{~d} y \leq \frac{1}{y_{0} \Phi(S) / S-\Phi(R)} I_{1, n}(s), \quad s \in[0, T] .
$$

Given $\delta>0$ we next define

$$
\mathcal{E}_{\delta}^{n, R}(t):=\sup \left\{\int_{E} u_{n}(t, y) \mathrm{d} y ; E \subset\left(y_{0}, R\right) \text { measurable, }|E| \leq \delta\right\}
$$

and show that

$$
\lim _{\delta \rightarrow 0} \sup _{\substack{n \in \mathbb{N} \\ t \in[0, T]}} \mathcal{E}_{\delta}^{n, R}(t)=0 .
$$

Introducing with $\mathbb{U}_{n}(t, s), 0 \leq s \leq t \leq \infty$, the (positive) evolution operator on $L_{1}(Y)$ corresponding to the operator $-A_{n}(t):=-V_{n}(t) \partial_{y}(\tau \cdot)$ with $V_{n}(t):=v_{n}(t) /\left(1+\nu\left\|u_{n}(t)\right\|_{0}\right)$ we first note that we can write $u_{n}$ in the form

$$
u_{n}(t)=\mathbb{U}_{n}(t, 0) u_{n}^{0}+\int_{0}^{t} \mathbb{U}_{n}(t, s)\left(L_{n}\left[u_{n}(s)\right]+Q_{n}\left[u_{n}(s)\right]\right) \mathrm{d} s .
$$

Recall from [21, Lemma 4.1] that we have

$$
\sup _{\substack{E \subset\left(y_{0}, R\right),|E| \leq \delta}} \int_{E} \mathbb{U}_{n}(t, s) f \mathrm{~d} y \leq \sup _{\substack{F \subset\left(y_{0}, R\right),|F| \lambda_{R}(\delta)}} \int_{F} f \mathrm{~d} y
$$

for all $f \in L_{1}^{+}(Y)$, where

$$
\lambda_{R}^{n}(\delta):=\tau_{*} R \sup _{\substack{E \subset\left(y_{0}, R\right),|E| \leq \delta}} \int_{E} \frac{\mathrm{d} z}{\tau_{n}(z)} .
$$

Note that by (4.3)

$$
\lambda_{R}^{n}(\delta) \leq \frac{2 \tau_{*} R}{\tau_{0}} \delta=: \lambda_{R}(\delta)
$$


It then follows from (4.23)-(4.25) and the positivity of $u_{n}$ (i.e. neglecting negative contributions in (4.23) that

$$
\begin{aligned}
\mathcal{E}_{\delta}^{n, R}(t) \leq \mathcal{E}_{\lambda_{R}(\delta)}^{n, R}(0) & +2 \int_{0}^{t} \sup _{\substack{F \subset\left(y_{0}, R\right) \\
|F| \leq \lambda_{R}(\delta)}} \int_{y_{0}}^{\infty} u_{n}(s, y) \beta_{n}(y) \int_{y_{0}}^{y} \mathbf{1}_{F}(z) \kappa(z, y) \mathrm{d} z \mathrm{~d} y \mathrm{~d} s \\
& +\int_{0}^{t} \sup _{\substack{F \subset\left(2 y_{0}, R\right) \\
|F| \leq \lambda_{R}(\delta)}} \int_{F} \int_{y_{0}}^{y-y_{0}} \eta_{n}(y-z, z) u_{n}(s, y-z) u_{n}(s, z) \mathrm{d} z \mathrm{~d} y \mathrm{~d} s .
\end{aligned}
$$

We now estimate the intergal terms on the right-hand side. First observe that, using (4.21), the second term on the right-hand side of (4.26) can be bounded above as

$$
2 \int_{0}^{t} \sup _{\substack{F \subset\left(y_{0}, R\right) \\|F| \leq \lambda_{R}(\delta)}} \int_{y_{0}}^{\infty} u_{n}(s, y) \beta_{n}(y) \int_{y_{0}}^{y} \mathbf{1}_{F}(z) \kappa(z, y) \mathrm{d} z \mathrm{~d} y \mathrm{~d} s \leq P(\delta, S)
$$

where

$$
\begin{aligned}
P(\delta, S):=2 \sup _{\substack{t \in[0, T] \\
n \in \mathbb{N}}}\left\{\int _ { 0 } ^ { t } \operatorname { s u p } _ { \substack { F \subset ( y _ { 0 } , R ) \\
| F | \leq \lambda _ { R } ( \delta ) } } \left(\int_{y_{0}}^{S} u_{n}(s, y) \beta_{n}(y) \int_{y_{0}}^{R \wedge y} \mathbf{1}_{F}(z) \kappa(z, y) \mathrm{d} z \mathrm{~d} y\right.\right. \\
\\
\left.\left.+\frac{1}{y_{0} \Phi(S) / S-\Phi(R)} I_{1, n}(s)\right) \mathrm{d} s\right\} .
\end{aligned}
$$

As for the last term on the right-hand side of (4.26) we fix a measurable subset $F$ of $\left(2 y_{0}, R\right)$ with measure $|F| \leq \lambda_{R}(\delta)$ and $s \in[0, T]$. Then we deduce first from (4.5) and then from (4.9) along with the translation invariance of the Lebesgue measure that

$$
\begin{aligned}
\int_{F} \int_{y_{0}}^{y-y_{0}} \eta_{n}( & -z, z) u_{n}(s, y-z) u_{n}(s, z) \mathrm{d} z \mathrm{~d} y \\
= & \int_{y_{0}}^{\infty} u_{n}(s, z) \int_{y_{0}}^{\infty} \mathbf{1}_{F}(y+z) \eta_{n}(y, z) u_{n}(s, y) \mathrm{d} y \mathrm{~d} z \\
\leq & c(R) \int_{y_{0}}^{\infty} u_{n}(s, z) \int_{y_{0}}^{\infty} \mathbf{1}_{-z+F}(y) u_{n}(s, y) \mathrm{d} y \mathrm{~d} z \\
& \leq c(R, T) \mathcal{E}_{\lambda_{R}(\delta)}^{n, R}(s) .
\end{aligned}
$$

Since clearly $\mathcal{E}_{\lambda_{R}(\delta)}^{n, R}(s) \leq c(R) \mathcal{E}_{\delta}^{n, R}(s)$ due to the definition of $\lambda_{R}(\delta)$ we obtain

$$
\int_{0}^{t} \sup _{\substack{F \subset\left(2 y_{0}, R\right) \\|F| \leq \lambda_{R}(\delta)}} \int_{F} \int_{y_{0}}^{y-y_{0}} \eta_{n}(y-z, z) u_{n}(s, y-z) u_{n}(s, z) \mathrm{d} z \mathrm{~d} y \mathrm{~d} s \leq c(R, T) \int_{0}^{t} \mathcal{E}_{\delta}^{n, R}(s) \mathrm{d} s .
$$

Therefore, combining (4.26)-(4.28) we deduce that

$$
\mathcal{E}_{\delta}^{n, R}(t) \leq c(R) \mathcal{E}_{\delta}^{n, R}(0)+P(\delta, S)+c(R, T) \int_{0}^{t} \mathcal{E}_{\delta}^{n, R}(s) \mathrm{d} s
$$

and hence, applying Gronwall's inequality observing that $\mathcal{E}_{\delta}^{n, R} \in C\left([0, T], \mathbb{R}^{+}\right)$,

$$
\mathcal{E}_{\delta}^{n, R}(t) \leq c(R, T)\left(\mathcal{E}_{\delta}^{n, R}(0)+P(\delta, S)\right), \quad t \in[0, T], \quad n \in \mathbb{N}, \quad \delta>0,
$$

provided that $S>R>2 y_{0}$. Noticing then that, on the one hand, (4.2) and the Dunford-Pettis Theorem [4, Theorem 4.21.2] imply

$$
\lim _{\delta \rightarrow 0} \sup _{n \in \mathbb{N}} \mathcal{E}_{\delta}^{n, R}(0)=\lim _{\delta \rightarrow 0} \sup _{n \in \mathbb{N}} \sup _{\substack{F \subset\left(y_{0}, R\right) \\|F| \leq \delta}} \int_{F} u_{n}^{0}(y) \mathrm{d} y=0,
$$


and, on the other hand, that $P(\delta, S)$ can be made arbitrarily small by choosing first $S>R$ large and then $\delta$ small enough according to (2.15), (4.1), (4.9), and (4.13), we deduce that

$$
\lim _{\delta \rightarrow 0} \sup _{n \in \mathbb{N}} \mathcal{E}_{\delta}^{n, R}(t)=0,
$$

uniformly with respect to $t \in[0, T]$ and for every $R>y_{0}$. Combining (4.20) and (4.29), the existence of a weakly compact subset $K_{T}$ of $L_{1}(Y, y \mathrm{~d} y)$ such that $u_{n}(t) \in K_{T}$ for $n \in \mathbb{N}$ and $0 \leq t \leq T$ is then a consequence of the Dunford-Pettis Theorem.

Finally, the estimate (4.19) is derived exactly as in [13, Lemma 4.1] owing to assumptions (2.13), (2.16), the properties of $\Phi$ and the bounds (4.9), (4.13).

Lemma 4.5. The family $\left\{u_{n} ; n \in \mathbb{N}\right\}$ is weakly equicontinuous in $L_{1}(Y, y \mathrm{~d} y)$ at every $t \in[0, T]$.

Proof. This follows along the lines of part (iii) of the proof of [19, Theorem 4.3] by using [4.9), (4.21), and the weak compactness of $\left\{u_{n}(t) ; t \in[0, T], n \in \mathbb{N}\right\}$ in $L_{1}(Y, y \mathrm{~d} y)$ shown in Proposition 4.4

Lemma 4.6. The family $\left\{v_{n} ; n \in \mathbb{N}\right\}$ is relatively compact in $C([0, T])$.

Proof. This is a consequence of Proposition 4.4 and can be shown exactly as in [13, Lemma 4.3] by testing the truncated equation (1.2) by $\varphi(y)=y$ and additionally observing that

$$
\int_{y_{0}}^{\infty} Q_{n}\left[u_{n}\right](y) y \mathrm{~d} y=0 \text {. }
$$

4.3. Proof of Theorem 2.3. We are now in a position to prove Theorem 2.3. It follows from Proposition 4.4. Lemma 4.5, Lemma 4.6 and a variant of the Arzelà-Ascoli Theorem [20, Theorem 1.3.2] that there are subsequences (not relabeled) $\left(v_{n}\right),\left(u_{n}\right)$ and functions $v \in C\left(\mathbb{R}^{+}\right), u \in C\left(\mathbb{R}^{+}, L_{1, \mathrm{w}}(Y, y \mathrm{~d} y)\right)$ such that

$$
\begin{aligned}
& v_{n} \rightarrow v \quad \text { in } \quad C([0, T]), \\
& u_{n} \rightarrow u \quad \text { in } \quad C\left([0, T], L_{1, \mathrm{w}}(Y, y \mathrm{~d} y)\right)
\end{aligned}
$$

for each $T>0$. In addition, $v(t) \geq 0$ and $u(t) \geq 0$. It remains to show that $(v, u)$ is a weak solution to (1.1)-(1.2). Since $\left(v_{n}, u_{n}\right)$ satisfies the weak formulation given in Definition 2.2 we pass to the limit in each of the corresponding terms. This is rather standard by now and except for the bilinear polymer joining terms similar to [13]. Indeed, using Fatou's Lemma we infer from (4.19) and 4.31] that

$$
[(t, y) \mapsto \beta(y) u(t, y)] \in L_{1}((0, T) \times Y),
$$

while (4.31) and (2.17) clearly imply that

$$
[(t, y, z) \mapsto \eta(y, z) u(t, z) u(t, y)] \in L_{1}((0, T) \times Y \times Y) .
$$

Also, (4.1), (4.14), and (4.31) ensure that

$$
\lim _{n \rightarrow \infty} \int_{0}^{t} \int_{y_{0}}^{\infty} y \mu_{n}(y) u_{n}(s, y) \mathrm{d} y \mathrm{~d} s=\int_{0}^{t} \int_{y_{0}}^{\infty} y \mu(y) u(s, y) \mathrm{d} y \mathrm{~d} s<\infty
$$

for any fixed $t \in[0, T]$. For $\varphi \in W_{\infty}^{1}(Y)$ it follows then from (2.14), (4.3), (4.4), (4.20), (4.30), (4.31) that

$$
\begin{aligned}
\lim _{n \rightarrow \infty} \int_{0}^{t} \frac{v_{n}(s)}{1+\nu\left\|u_{n}(s)\right\|_{0}} & \int_{y_{0}}^{\infty} \varphi^{\prime}(y) \tau_{n}(y) u_{n}(s, y) \mathrm{d} y \mathrm{~d} s \\
& =\int_{0}^{t} \frac{v(s)}{1+\nu\|u(s)\|_{0}} \int_{y_{0}}^{\infty} \varphi^{\prime}(y) \tau(y) u(s, y) \mathrm{d} y \mathrm{~d} s .
\end{aligned}
$$


Therefore, using (2.4), (2.13), (4.1), (4.13), (4.21), (4.31), and (4.32) it readily follows that

$$
\lim _{n \rightarrow \infty} \int_{0}^{t} \int_{y_{0}}^{\infty} \varphi(y) \mu_{n}(y) u_{n}(s, y) \mathrm{d} y \mathrm{~d} s=\int_{0}^{t} \int_{y_{0}}^{\infty} \varphi(y) \mu(y) u(s, y) \mathrm{d} y \mathrm{~d} s
$$

and

$$
\begin{aligned}
& \lim _{n \rightarrow \infty} \int_{0}^{t} \int_{y_{0}}^{\infty} u_{n}(s, y) \beta_{n}(y)\left(-\varphi(y)+2 \int_{y_{0}}^{y} \varphi(z) \kappa(z, y) \mathrm{d} z\right) \mathrm{d} y \mathrm{~d} s \\
&=\int_{0}^{t} \int_{y_{0}}^{\infty} u(y) \beta(y)\left(-\varphi(y)+2 \int_{y_{0}}^{y} \varphi(z) \kappa(z, y) \mathrm{d} z\right) \mathrm{d} y \mathrm{~d} s
\end{aligned}
$$

for any compactly supported test function $\varphi \in W_{\infty}^{1}(Y)$, say with support $\left[y_{0}, R\right]$, by observing that $\mu_{n}=\mu$ and $\beta_{n}=\beta$ on $\left[y_{0}, R\right]$ when $n$ is so large that $\mathcal{S}_{n}(T)>R$ (see (4.8)). For such a test function $\varphi$ one then also shows based on (2.17), (4.7), (4.9), (4.20), and (4.31) that

$$
\begin{aligned}
\lim _{n \rightarrow \infty} \int_{0}^{t} \int_{y_{0}}^{\infty} \int_{y_{0}}^{\infty} \varphi(y+z) & \eta_{n}(y, z) u_{n}(s, z) u_{n}(s, y) \mathrm{d} z \mathrm{~d} y \mathrm{~d} s \\
= & \int_{0}^{t} \int_{y_{0}}^{\infty} \int_{y_{0}}^{\infty} \varphi(y+z) \eta(y, z) u(s, z) u(s, y) \mathrm{d} z \mathrm{~d} y \mathrm{~d} s
\end{aligned}
$$

and

$$
\begin{aligned}
& \lim _{n \rightarrow \infty} \int_{0}^{t} \int_{y_{0}}^{\infty} \int_{y_{0}}^{\infty} \varphi(y) \eta_{n}(y, z) u_{n}(s, z) u_{n}(s, y) \mathrm{d} z \mathrm{~d} y \mathrm{~d} s \\
&=\int_{0}^{t} \int_{y_{0}}^{\infty} \int_{y_{0}}^{\infty} \varphi(y) \eta(y, z) u(s, z) u(s, y) \mathrm{d} z \mathrm{~d} y \mathrm{~d} s .
\end{aligned}
$$

A classical truncation argument along with (4.32)-(4.34) then entails that (4.36)-(4.39) hold true for any test function $\varphi \in W_{\infty}^{1}(Y)$. Consequently, $u$ satisfies the weak formulation and it similarly follows from (4.13), (4.9), (4.30), (4.31) that $v$ satisfies equation (1.1) and $v(t)>0, t \in[0, T]$.

Finally, (4.30), (4.31), and (4.34) guarantee that (2.8) also holds for $(v, u)$. This proves Theorem 2.3

4.4. Proof of Proposition 2.4. Let now $\theta=\alpha+\rho \leq 1$ and suppose that $u^{0} \in L_{1}^{+}\left(Y, y^{\sigma} \mathrm{d} y\right)$ for some $\sigma \geq 1$. Let $t \in[0, T]$. Since $u_{n}(t, \cdot)$ is compactly supported we may test (1.2) by $\varphi(y)=y^{\sigma}$ and obtain from (2.6) and (2.7)

$$
\begin{aligned}
\frac{\mathrm{d}}{\mathrm{d} t} \int_{y_{0}}^{\infty} y^{\sigma} u_{n}(t, y) \mathrm{d} y= & \sigma v_{n}(t) \int_{y_{0}}^{\infty} y^{\sigma-1} \tau_{n}(y) u_{n}(t, y) \mathrm{d} y-\int_{y_{0}}^{\infty} y^{\sigma}\left(\mu_{n}(y)+\beta_{n}(y)\right) u_{n}(t, y) \mathrm{d} y \\
& +2 \int_{y_{0}}^{\infty} u_{n}(s, y) \beta_{n}(y) \int_{y_{0}}^{y} z^{\sigma} \kappa(z, y) \mathrm{d} z \mathrm{~d} y \\
& +\int_{y_{0}}^{\infty} \int_{y_{0}}^{\infty}\left((y+z)^{\sigma}-y^{\sigma}-z^{\sigma}\right) \eta_{n}(y, z) u_{n}(t, y) u_{n}(t, z) \mathrm{d} z \mathrm{~d} y .
\end{aligned}
$$

Note that (2.3) entails

$$
2 \int_{y_{0}}^{y} z^{\sigma} \kappa(z, y) \mathrm{d} z \leq y^{\sigma}, \quad y>y_{0}
$$

while (4.5) implies

$$
\left((y+z)^{\sigma}-y^{\sigma}-z^{\sigma}\right) \eta_{n}(y, z) \leq c(\sigma) y^{\sigma-1} z \eta_{n}(y, z) \leq c y^{\sigma} z, \quad y_{0} \leq z \leq y,
$$

so that, according to 4.9 ,

$$
\int_{y_{0}}^{\infty} \int_{y_{0}}^{\infty}\left((y+z)^{\sigma}-y^{\sigma}-z^{\sigma}\right) \eta_{n}(y, z) u_{n}(t, y) u_{n}(t, z) \mathrm{d} z \mathrm{~d} y \leq c(T) \int_{y_{0}}^{\infty} y^{\sigma} u_{n}(t, y) \mathrm{d} y .
$$


Hence we derive from (4.3) that

$$
\frac{\mathrm{d}}{\mathrm{d} t} \int_{y_{0}}^{\infty} y^{\sigma} u_{n}(t, y) \mathrm{d} y \leq c(T) \int_{y_{0}}^{\infty} y^{\sigma} u_{n}(t, y) \mathrm{d} y
$$

and consequently

$$
\left\|u_{n}(t)\right\|_{L_{1}\left(Y, y^{\sigma} \mathrm{d} y\right)} \leq c(T), \quad t \in[0, T], \quad n \in \mathbb{N} .
$$

Since this estimate is preserved for $u$ due to 4 4.31), Proposition 2.4 follows.

\section{REFERENCES}

[1] V. Calvez, N. Lenuzza, M. Doumic, J.-P. Deslys, F. Mouthon, B. Perthame. Prion dynamics with size dependency-strain phenomena. J. Biol. Dyn. 4 (2010), no. 1, 28-42.

[2] V. Calvez, N. Lenuzza, D. Oelz, J.-P. Deslys, P. Laurent, F. Mouthon, B. Perthame. Size distribution dependence of prion aggregates infectivity. Math. Biosci. 217 (2009), no. 1, 89-99.

[3] M. Doumic, P. Gabriel. Eigenelements of a general aggregation-fragmentation model. Math. Models Methods Appl. Sci. 20 (2010), 757-783.

[4] R.E. Edwards. Functional Analysis. Theory and Applications. Dover Publications, Inc., New York, 1995.

[5] H. Engler, J. Prüss, G. F. Webb. Analysis of a model for the dynamics of prions II. J. Math. Anal. Appl. 324 (2006), 98-117.

[6] M. Escobedo, Ph. Laurençot, S. Mischler, B. Perthame. Gelation and mass conservation in coagulation-fragmentation models. J. Differential Equations 195 (2003), 143-174.

[7] P. Gabriel. Global stability for the prion equation with general incidence. Math. Biosci. Eng. 12 (2015), no. 4, $789-801$.

[8] M. L. Greer, P. van den Driessche, L. Wang, G. F. Webb. Effects of general incidence and polymer joining on nucleated polymerzation in a model of prion proliferation. SIAM J. Appl. Math. 68 (2007), no. 1, 154-170.

[9] M. L. Greer, L. Pujo-Menjouet, G. F. Webb. A mathematical analysis of the dynamics of prion proliferation. J. Theoret. Biol. 242 (2006), 598-606.

[10] C.-H. Lê. Etude de la classe des opérateurs m-accrétifs de $L^{1}(\Omega)$ et accrétifs dans $L^{\infty}(\Omega)$. Thèse de 3-ème cycle. Université de Paris VI, Paris, 1977.

[11] Ph. Laurençot. On a class of continuous coagulation-fragmentation equations. J. Differential Equations 167 (2000), $245-274$.

[12] Ph. Laurençot. The Lifshitz-Slyozov equation with encounters. Math. Models Methods Appl. Sci. 11 (2001) 731-748.

[13] $\mathrm{Ph}$. Laurençot, Ch. Walker. Well-posedness for a model of prion proliferation dynamics. J. Evol. Equ. 7 (2007), 241-264.

[14] E. Leis, Ch. Walker. Uniqueness of weak solutions to a prion equation with polymer joining. Preprint (2016).

[15] J. Masel, V. A. A. Jansen, M. S. Nowak. Quantifying the kinetic parameters of prion replication. Biophys. Chem. 77 (1999), $139-152$.

[16] M. A. Nowak, D. C. Krakauer, A. Klug, R. M. May. Prion infection dynamics. Integr. Biol. 1 (1998), 3-15.

[17] A. Pazy. Semigroups of Linear Operatos and Applications to Partial Differential Equations. Springer, Berlin, New York, Heidelberg, 1983.

[18] J. Prüss, L. Pujo-Menjouet, G. F. Webb, R. Zacher. Analysis of a model for the dynamics of prions. Discrete Contin. Din. Syst. Ser. B 6 (2006), 225-235.

[19] G. Simonett, Ch. Walker. On the solvability of a mathematical model for prion proliferation. J. Math.Anal. Appl. 324 (2006), 580-603.

[20] I. I. Vrabie. Compactness Methods for Nonlinear Evolutions, second ed. Longman Scientific \& Technical, Harlow; copublished in the United States with John Wiley \& Sons, Inc., New York, 1995.

[21] Ch. Walker. Prion proliferation with unbounded polymerization rates. Proceedings of the Sixth Mississippi State-UBA Conference on Differential Equations and Computational Simulations, 387-397, Electron. J. Differ. Equ. Conf., 15, Southwest Texas State Univ., San Marcos, TX, 2007.

Leibniz Universität Hannover, Institut FÜr Angewandte Mathematik, Welfengarten 1, D-30167 HanNOVER, GERMANY

E-mail address: leis@ifam.uni-hannover.de

Leibniz Universität Hannover, Institut für Angewandte Mathematik, Welfengarten 1, D-30167 HanNOVER, GERMANY

E-mail address: walkereifam.uni-hannover.de 